University of Zurich

Department of Economics

Working Paper Series

ISSN 1664-7041 (print)

ISSN 1664-705X (online)

Working Paper No. 193

\title{
On the Optimal Quantity of Liquid Bonds
}

Samuel Huber and Jaehong Kim

Revised version, April 2017 


\title{
On the Optimal Quantity of Liquid Bonds*
}

\author{
Samuel Huber \\ University of Basel
}

\author{
Jaehong Kim \\ Xiamen University
}

April 6, 2017

\begin{abstract}
We develop a dynamic general equilibrium model to analyze the optimal quantity of liquid bonds by investigating the following three questions: Under what conditions is it socially desirable to contract the bond supply, what incentive problems are mitigated by doing this, and how large are the effects? We show that reducing the bond supply induces agents to increase their demand for money, which can enhance welfare by improving the allocation of the medium of exchange. However, this effect fails for high inflation rates, because agents hold so little money in the first place that manipulating the bond supply is not enough to correct the misallocation.
\end{abstract}

Keywords: Monetary theory, over-the-counter markets, bond supply, financial intermediation, money demand, pecuniary externality.

JEL Classification: D52, D62, E31, E40, E50, G11, G12, G28.

\section{Introduction}

Governments generally issue two intrinsically useless objects: money and bonds. While money is perfectly liquid, divisible, and widely accepted as a medium of exchange, bonds are "intentionally handicapped (hence discounted)" due to "physical or legal characteristics that render them less liquid than money" (Andolfatto, 2011, p.133). By conducting monetary policy, central banks have a major control over the composition of these issued objects.

In this paper, we take a closer look at optimal quantity of liquid bonds. That is, we analyze under what conditions it can be desirable to contract the bond supply, what incentive problems this mitigates, and how large these effects are.

${ }^{*}$ We would like to thank the editor and two anonymous referees for very helpful comments. We also thank the Fundamental Research Funds for the Central Universities for financial support, Grant 20720171070. Samuel Huber is a research fellow at the Department of Economic Theory, University of Basel. E-mail: samuel_h@gmx.ch. Jaehong Kim is an associate professor at the Wang Yanan Institute for Studies in Economics and the School of Economics, Xiamen University. E-mail: jaehongkim@xmu.edu.cn. 
For this purpose, we construct a microfounded monetary model, where trading in financial markets is essential; i.e., trading improves the allocation of the medium of exchange. In our model, agents face idiosyncratic liquidity shocks, and they hold a portfolio composed of money and government bonds. Money can be directly used to purchase goods and thus serves as a medium of exchange. In contrast, government bonds cannot be used as a medium of exchange, but are a superior store of value. ${ }^{1}$ The idiosyncratic liquidity shocks generate an ex-post inefficient allocation of the medium of exchange: Some agents will hold money, but have no current need for it, while other agents will hold insufficient amounts of money to satisfy their liquidity needs. A secondary financial market allows agents to trade money for bonds and so improves the allocation of the medium of exchange. The secondary financial market is an over-the-counter market, that embeds the recent advances in search theory. We assume that the monetary authority directly controls the bond-to-money ratio and thereby the supply of liquid government bonds.

We derive our results in the monetary steady state equilibrium; i.e., there are no aggregate shocks. Furthermore, we focus on the optimal bond supply in an economy, where the efficient allocation is not attainable; i.e., on an economy with inflation rates above the Friedman rule. Our main finding is that contracting the bond supply mitigates a pecuniary externality and so improves the allocation and welfare. The existence of this externality causes the equilibrium to be inefficient, such that government interventions can be welfare-improving. ${ }^{2}$ In our model, the secondary financial market reduces the incentive to self-insure against liquidity shocks, and agents attempt to benefit from money held by other market participants. As a result, the aggregate demand for money is too low, and contracting the bond supply can mitigate this externality. This is because contracting the supply makes bonds scarce and increases their price above their fundamental value. In turn, agents increase their demand for money, which marginally increases the value of money and so improves the insurance for all market participants.

We show that the optimal supply of bonds critically depends on the over-the-counter structure of the secondary bond market. If the market features high bargaining frictions, the optimal bond supply is large, whereas if the market is very competitive, the opposite is true. For a fully competitive market, it is even optimal to reduce the bond supply to zero. We also show that contracting the bond supply is only socially beneficial for low inflation rates. The reason is that for higher inflation rates, the demand for money is so low that contracting the bond supply is not sufficient to improve the allocation.

\section{Literature}

Our paper is in the field of the "New Monetarist Economics," a branch of literature that builds on Kiyotaki and Wright (1989) and especially Lagos and Wright (2005). ${ }^{3}$ In our model, agent-

\footnotetext{
${ }^{1}$ It is socially beneficial that government bonds cannot be used as a medium of exchange. Otherwise, bonds would be perfect substitutes for money and thus be redundant. See Kocherlakota (2003), Andolfatto (2011), Berentsen and Waller (2011), and Berentsen et al. (2014) for a more detailed discussion.

${ }^{2}$ See Greenwald and Stiglitz (1986) and Berentsen et al. (2016) for a more detailed discussion.

${ }^{3}$ A detailed overview of major contributions to this field can be found in Williamson and Wright (2010), Nosal and Rocheteau (2011), and Lagos et al. (2015).
} 
types are alternating, which generates an ex-post inefficient allocation of money and generates an endogenous role for a financial market, where agents can adjust their portfolios. In this sense, our paper is related to Berentsen et al. (2007) and Berentsen and Waller (2011). In contrast to these studies, we do not assume competitive pricing in the secondary financial market and model the exchange process in more detail. Concretely, we assume over-the-counter trading with search and bargaining frictions in the spirit of Duffie et al. (2005). ${ }^{4}$

Unlike the above-mentioned studies, our main focus is on the optimal quantity of bonds and its implications for the allocation of money and welfare. More closely related to what we do are the studies by Williamson $(2012,2015,2016)$. While we assume that only money can be used as a medium of exchange, Williamson assumes that also claims to bonds are acceptable in some meetings. That is, Williamson assumes that agents do not directly trade money for bonds, but rather that banks collateralize their deposits with bonds and that agents can trade these claims for goods in monitored meetings. ${ }^{5}$ In the exchange process, Williamson assumes take-itor-leave-it offers, while we assume over-the-counter trading with search and bargaining frictions. This differentiation has interesting implications regarding the quantity of bonds, which are not present under the assumption of take-it-or-leave-it offers. Williamson models some features in more detail than we do, such as financial intermediation and the issuance of private debt, while we focus more explicitly on other issues. That is, we show under what conditions central banks succeed in controlling short-term interest rates by contracting the bond supply and when such a policy measure is socially beneficial. To do this, we do not solely focus on equilibria where bonds are scarce and exhibit a liquidity premium, but also focus on equilibria where bonds are plentiful, which allows us to obtain interesting insights. ${ }^{6}$ For instance, we find that in equilibria where bonds and money are plentiful, welfare can always be improved by contracting the bond supply. Furthermore, we find that a contraction of the bond supply is not socially beneficial if inflation is too high. Both arguments are missing in the studies by Williamson.

Gertler and Karadi (2013) present a model which is similar to Williamson (2012, 2015, 2016) in order to analyze the effects of central bank purchases of long-maturity government bonds or private loans. They find that central banks, as opposed to private intermediaries, obtain funds elastically; i.e., they can fund the purchase of long-term securities by issuing short-term debt. This provides central banks with a channel for large-scale asset purchases to be effective in reducing borrowing costs if financial assets are scarce. In contrast to Gertler and Karadi (2013), we focus on the optimal quantity of short-maturity bonds in order to control the short-term

\footnotetext{
${ }^{4}$ There is a rapidly growing literature which builds on the seminal contribution of Duffie et al. (2005). See, for instance, Ashcraft and Duffie (2007), Duffie et al. (2008), Lagos and Rocheteau (2009), Lagos et al. (2011), Rocheteau and Wright (2013), Lagos and Zhang (2015), Berentsen et al. (2016), Mattesini and Nosal (2016), Trejos and Wright (2016), and Geromichalos and Herrenbrueck (2016a, b).

${ }^{5}$ A more detailed discussion about the acceptability of illiquid assets can be found in Shi (2008), Lagos and Rocheteau (2008), Lester et al. (2012), Hu and Rocheteau (2013), and Rocheteau et al. (2015). Collateralization is discussed in Ferraris and Watanabe (2008), He et al. (2015), Li and Li (2012), Gu et al. (2013), Bethune et al. (2014), Andolfatto et al. (2015), and Rocheteau et al. (2015).

${ }^{6}$ The existence of liquidity premia is discussed in Geromichalos et al. (2007), Lagos and Rocheteau (2008), Lagos (2010a, b, 2011), Jacquet and Tan (2012), Lester et al. (2012), Nosal and Rocheteau (2013), Lagos and Zhang (2015), Berentsen et al. (2014, 2016), and Dominguez and Gomis-Porqueras (2016).
} 
interest rates. Furthermore, we focus in more detail on the societal implications of such a policy measure.

Similar to what we do, Herrenbrueck (2014) integrates an over-the-counter market in the spirit of Duffie et al. (2005) into a New Monetarist framework. Like Williamson (2012, 2015, 2016), Herrenbrueck assumes take-it-or-leave-it offers in the secondary financial market, while we assume that agents bargain over the terms of trade in bilateral meetings. Herrenbrueck finds that central bank purchases of government bonds are temporarily welfare-improving, because they transfer wealth to agents who value it more; i.e., to agents who want to sell financial assets for money in order to consume. Opposed to Herrenbrueck, we find that the main welfare-improving aspect of such a policy measure is that it corrects a pecuniary externality, and not that it transfers wealth to agents who value it more. In other words, it is not the resulting liquidity premium on bonds that improves welfare, but the incentive to increase the demand for money.

Along the same lines, Dominguez and Gomis-Porqueras (2016) analyze the effects of both passive and active fiscal and monetary policies. They find that due to the presence of a secondary financial market, multiple equilibria may occur. This happens in an environment where government bonds exhibit a liquidity premium, such that fiscal and monetary polciy are not independent of each other. The authors show that active monetary policy is likely to restore the uniqueness of the monetary steady state, independent of the fiscal policy. In contrast to Dominguez and Gomis-Porqueras (2016), we abstract from government expenditures and analyze the optimal quantity of liquid government bonds and its implications on the allocation and welfare.

Most closely related to our study is the work by Rocheteau et al. (2015). Similar to Williamson $(2012,2015,2016)$, the authors assume that bonds are accepted as a medium of exchange in some meetings, while they are not in others. That is, Rocheteau et al. (2015) focus on the acceptability and pledgability of bonds in an equilibrium where bonds are scarce, while we simplify according to this rationale and model the exchange process in more detail in order to analyze the welfare implications of contracting the bond supply. In contrast to us, Rocheteau et al. (2015) do not model a secondary financial market explicitly. Consequently, they cannot evaluate the role of search and bargaining frictions in secondary bond markets. Since search frictions are empirically well documented in the market for U.S. government bonds, we believe that it is important to incorporate these frictions in order to analyze the social implications of a contraction in the bond supply. ${ }^{7}$ Doing so allows us to show that search frictions are essential for a reduction in the bond supply to be welfare-improving. Furthermore, we do not solely focus on equilibria where bonds are scarce, which allows us to state that reducing the bond supply is only welfare-improving for low inflation rates.

Our paper is also related to the literature that focuses on correcting pecuniary externalities, such as Berentsen et al. (2014, 2016), and Boel and Waller (2015). Berentsen et al. (2014) find that adding search frictions to a competitive secondary financial market can be welfare-improving for high inflation rates. The reason is that adding search frictions increases the demand for money,

\footnotetext{
${ }^{7}$ See Duffie et al. (2005) and Krishnamurthy (2002) for a more detailed discussion about search frictions in the market for U.S. government bonds.
} 
which is welfare-improving, but it also increases consumption variability, since only some agents have access to the secondary financial market. In a similar framework to ours, Berentsen et al. (2016) find that the demand for money is too low in an equilibrium where trading is unconstrained and that imposing a financial transaction tax can correct this externality. However, imposing a financial transaction tax requires the central bank to operate the secondary financial market in order to perfectly enforce tax payment. In contrast, controlling the supply of liquid bonds is a conventional policy tool of central banks and it is crucial to understand the economic mechanisms behind such a policy instrument.

Using a similar framework like Rocheteau et al. (2015), Boel and Waller (2015) analyze the efficacy of central bank purchases of government bonds and private debt at the zero lower bound. In contrast to previous studies, Boel and Waller (2015) assume that the interventions of the central bank are only temporary and not permanent. They find that central bank purchases of government bonds can be beneficial at the zero lower bound, because they succeed in temporarily correcting a pecuniary externality. Their result, however, only holds under the condition that agents are heterogeneous in their preferences about future consumption, such that the Friedman rule fails to attain the first-best allocation. In contrast to Boel and Waller (2015), we analyze the optimal quantity of bonds in general and show under what conditions a contraction in the supply of bonds succeeds in implementing an interest rate target, such as the zero lower bound, and when such a policy measure is desirable from a societal point of view.

\section{Environment}

A $[0,1]$-continuum of agents live forever in discrete time. In each period, there are three markets that open sequentially. ${ }^{8}$ The first market is a secondary bond market, where agents trade money for nominal bonds. The second market is a goods market, where agents produce or consume market-2 goods. The third market is a centralized market, where all agents consume and produce market-3 goods, and financial contracts are redeemed. This market is called the primary bond market. All goods are perfectly divisible and non-storable.

At the beginning of each period, agents receive an idiosyncratic i.i.d. preference shock that determines whether they are producers or consumers in the goods market. With probability $n$, an agent can produce but not consume, and with probability $1-n$, he can consume but not produce. In the goods market, trading is competitive; i.e., agents trade against the market and take prices as given. Consumers gain utility $u(q)$ from $q$ consumption, where $u^{\prime}(q),-u^{\prime \prime}(q)>0$, $u^{\prime}(0)=\infty$, and $u^{\prime}(\infty)=0$. Producers incur a utility cost $c(q)=q$ from producing $q$ units of market-2 goods.

In the primary bond market, trading is perfectly frictionless and competitive. The market-3 good is produced and consumed by all agents using a linear production technology; i.e., $h$ units

\footnotetext{
${ }^{8}$ Our framework is similar to Berentsen et al. (2016), which builds on Lagos and Wright (2005). However, the contribution of Berentsen et al. (2016) is different. In particular, the authors investigate the social benefits of a financial transaction tax on bond transactions in an equilibrium where trading is unconstrained.
} 
of time produce $h$ units of goods. ${ }^{9}$ Agents gain utility $U(x)$ from $x$ consumption, where $U^{\prime}(x)$, $-U^{\prime \prime}(x)>0, U^{\prime}(0)=\infty$, and $U^{\prime}(\infty)=0$. Agents discount between periods with the discount factor $\beta$. A central bank operates in the primary bond market and issues two perfectly divisible and storable assets: money and one-period bonds. Both assets are intrinsically useless. Bonds are issued at a discount, and pay off one unit of money in the next-period primary bond market. Bonds are intangible objects; i.e., no physical object exists. In the goods market, agents cannot commit, and there is a lack of record-keeping. These two frictions imply that producers ask for immediate compensation from consumers. As bonds are intangible objects, only money can serve as a medium of exchange in the goods market. ${ }^{10}$ The per-capita stock of money is denoted by $M_{t}$, and the per-capita stock of newly issued bonds is denoted by $B_{t}$ at the end of period $t$. The issuance price of bonds in the primary bond market is denoted by $\rho_{t}$. Thus, the change in the stock of money in period $t$ is given by

$$
M_{t}-M_{t-1}=\tau_{t} M_{t-1}+B_{t-1}-\rho_{t} B_{t},
$$

which is determined by three components: the lump-sum money injections, $T_{t}=\tau_{t} M_{t-1}$, the money created to redeem previously issued bonds, $B_{t-1}$, and the money withdrawal from selling $B_{t}$ units of bonds at price $\rho_{t}$. We assume that there is a strictly positive initial stock of money $M_{0}$ and bonds $B_{0}$, where $B_{0} / M_{0}=\mathcal{B}$ denotes the bond-to-money ratio. For $\tau_{t}<0$, the central bank must be able to extract money via lump-sum taxes from the economy.

At the beginning of each period, and after the realization of the idiosyncratic preference shock, agents can trade money for bonds in the secondary bond market. Consumers and producers meet at random in bilateral meetings according to a reduced-form matching function $\xi \mathcal{M}(n, 1-n)$, where the parameter $\xi$ determines the efficiency of the matching process (see e.g., Rocheteau and Weill, 2011). By assumption, the matching function has constant returns to scale, and is continuous and increasing with respect to each of its arguments. The probability that a consumer meets a producer is denoted by $\delta=\xi \mathcal{M}(n, 1-n)(1-n)^{-1}$, and the probability that a producer meets a consumer is denoted by $\delta^{p}=\xi \mathcal{M}(n, 1-n) n^{-1}=\delta(1-n) n^{-1}$. Once in a meeting, agents bargain over the quantity of money and bonds to be exchanged. Agents who are able to participate in this market are called active and those who are not are called passive.

\subsection{Efficient Allocation}

As a benchmark exercise, we present the allocation chosen by a social planner who dictates consumption and production. The planner treats all agents symmetrically, and his optimization problem is

\footnotetext{
${ }^{9}$ The assumption of quasi-linear preferences in the primary bond market results in a degenerate end-of-period distribution of money holdings, which makes the model tractable (see Lagos and Wright, 2005).

${ }^{10}$ The necessary assumptions that make money essential are discussed in more detail in Kocherlakota (1998), Wallace (2001), Lagos and Wright (2005) and Shi (2006). A more detailed discussion about why bonds cannot be used as a medium of exchange can be found in Kocherlakota (2003), Andolfatto (2011), Berentsen and Waller (2011), Rocheteau et al. (2015), and Berentsen et al. (2014, 2016). This assumption is crucial, since it allows us to break the irrelevance theorem proposed in Wallace (1981).
} 


$$
W=\max _{h, x, q}\left[(1-n) u(q)-n q_{p}\right]+U(x)-h,
$$

subject to the feasibility constraint $h \geq x$ and the market-clearing condition $n q_{p} \geq(1-n) q$. The efficient allocation satisfies $U^{\prime}\left(x^{*}\right)=1, u^{\prime}\left(q^{*}\right)=1$, and $h^{*}=x^{*}$.

\section{The Agent's Decisions}

For notational simplicity, we omit the time subscript $t$ going forward. Next-period variables are indexed by +1 , and previous-period variables by -1 . In what follows, we study the agents' decisions in a representative period $t$ and work backwards from the last market (primary bond market) to the first market (secondary bond market).

\subsection{Primary Bond Market}

In the primary bond market, agents can acquire any amount of money and newly issued bonds at price $\rho$. Agents want to hold money, because they will use it in the next-period goods market, if they turn out to be consumers. In contrast, bonds cannot be used as a medium of exchange; i.e., they are illiquid, but they can be traded for money in the next-period secondary bond market, which opens before the goods market. Furthermore, agents can produce and consume the market3 goods using a linear production technology; they receive money for maturing bonds; can trade money for market-3 goods; and they receive the lump-sum money transfer $T$ from the central bank. An agent entering the primary bond market with $m$ units of money and $b$ units of bonds has the value function $V_{3}(m, b)$. He solves the following decision problem:

$$
V_{3}(m, b)=\max _{x, h, m_{+1}, b_{+1}}\left[U(x)-h+\beta V_{1}\left(m_{+1}, b_{+1}\right)\right]
$$

subject to

$$
x+\phi m_{+1}+\phi \rho b_{+1}=h+\phi m+\phi b+\phi T,
$$

where $\phi$ is the price of money in terms of market-3 goods, and $h$ denotes hours worked. The first-order conditions with respect to $m_{+1}, b_{+1}$ and $x$ are $U^{\prime}(x)=1$, and

$$
\frac{\beta \partial V_{1}}{\partial m_{+1}}=\rho^{-1} \frac{\beta \partial V_{1}}{\partial b_{+1}}=\phi
$$

According to (5), the marginal cost of taking one additional unit of bonds into the next period, $\rho \phi$, is lower than that for money, $\phi$, for any $\rho<1$. The reason is that bonds are only beneficial to agents who will be active consumers in the next period. Therefore, bonds exhibit a lower marginal benefit than money for $\rho<1$, which is denoted by $\beta \partial V_{1} / \partial b_{+1}$. Due to the quasi-linear preferences, the choice of $m_{+1}$ and $b_{+1}$ is independent of $m$ and $b$. As a result, each agent exits the primary bond market with the same amount of money and bonds. The envelope conditions 
are

$$
\frac{\partial V_{3}}{\partial m}=\frac{\partial V_{3}}{\partial b}=\phi
$$

The above equation states that at the beginning of the primary bond market, the marginal value of money and bonds is equal to $\phi$. This is because bonds are redeemed at their face value in this market.

\subsection{Goods Market}

We assume competitive pricing in the goods market; i.e., all agents take prices as given and trade against the market. ${ }^{11}$ Consider the consumer's decision problem, where $p$ denotes the price of one unit of the market-2 good $q$ :

$$
V_{2}^{c}(m, b)=\max _{q}\left[\begin{array}{c}
u(q)+V_{3}(m-p q, b) \\
\text { s.t. } m \geq p q .
\end{array}\right] .
$$

The constraint states that a consumer cannot spend more money than the amount he brings into this market. If the constraint is non-binding, we have $\partial q / \partial m=0$ and $u^{\prime}(q)=1$. If the constraint is binding, we have $\partial q / \partial m=\phi$ and $u^{\prime}(q)>1$. In this case, the buyer's envelope conditions are

$$
\frac{\partial V_{2}^{c}}{\partial m}=\phi u^{\prime}(q) \quad \text { and } \quad \frac{\partial V_{2}^{c}}{\partial b}=\phi,
$$

where we have used the envelope conditions in the primary bond market.

The producer's value function in the goods market is

$$
V_{2}^{p}(m, b)=\max _{q_{p}}\left[-c\left(q_{p}\right)+V_{3}\left(m+p q_{p}, b\right)\right],
$$

where $q_{p}$ satisfies the market-clearing condition

$$
(1-n)[\delta \hat{q}+(1-\delta) q]=n q_{p}
$$

and where the symbol "^" denotes the quantities traded by active agents. The market-clearing condition states that the quantities consumed by active and passive consumers are different, since active consumers could adjust their portfolio in the secondary bond market and thus hold more money than passive consumers. According to (10), the produced quantity of producers, $n q_{p}$, equals the quantity consumed by active consumers, $(1-n) \delta \hat{q}$, plus the quantity consumed by passive consumers, $(1-n)(1-\delta) q$.

It is easy to see that $p \phi=c^{\prime}\left(q_{p}\right)=1$ holds in any monetary equilibrium. The reason is the following. For $p \phi<c^{\prime}\left(q_{p}\right)=1$, there is no trade, because it is suboptimal for producers to trade in the goods market. For $p \phi>c^{\prime}\left(q_{p}\right)=1$, each single producer has an incentive to sell more

\footnotetext{
${ }^{11}$ We have also derived our model for other trading protocols in the goods market, which feature search- and bargaining frictions; i.e., Nash bargaining and Kalai bargaining. Since changing the market structure only affected our results quantitatively, but not qualitatively, we do not present these results here.
} 
goods by (9). Hence, selling any finite amount of goods is suboptimal; i.e., the optimal strategy for producers is not supported by the market-clearing condition (10). Therefore, the equilibrium price must be $p \phi=c^{\prime}\left(q_{p}\right)=1$.

Taking the total derivative of (9) with respect to $m$ and $b$ and using (6) yields the envelope conditions of the producer:

$$
\frac{\partial V_{2}^{p}}{\partial m}=\frac{\partial V_{2}^{p}}{\partial b}=\phi
$$

Because producers cannot use money or bonds in this market, their marginal benefit equals the price of money in the primary bond market.

\subsection{Secondary Bond Market}

In the secondary bond market, the terms of trade are determined by the proportional bargaining solution proposed by Kalai (1977), which is increasingly popular in monetary economics due to its monotonicity properties. ${ }^{12}$ Consumers and producers are matched pairwise and bargain over the terms of trade. Consumers want to sell bonds for money in order to satisfy their consumption needs in the goods market. On the other hand, producers have no use for money in the goods market and are thus willing to sell money for bonds. Let $\left(m_{j}, b_{j}\right),\left(\hat{m}_{j}, \hat{b}_{j}\right)$ denote the portfolios of an active agent before and after trading in the secondary bond market, respectively. By the market-clearing condition, we have

$$
\hat{m}_{c}-m_{c}=-\left(\hat{m}_{p}-m_{p}\right) \quad \text { and } \quad \hat{b}_{p}-b_{p}=-\left(\hat{b}_{c}-b_{c}\right) .
$$

Let $d_{m} \equiv \hat{m}_{c}-m_{c}$ and $d_{b} \equiv \hat{b}_{p}-b_{p}$ be the trading amounts of money and bonds in the secondary bond market. Hence, we have the budget constraints for producers and consumers,

$$
\phi m_{p} \geq \phi d_{m} \quad \text { and } \quad \phi b_{c} \geq \phi d_{b},
$$

which state that producers cannot offer more money than they have, and consumers cannot offer more bonds than they have.

The Kalai constraint states that the trade surplus is split among producers and consumers according to their bargaining power. It is given by

$$
(1-\eta)\left[u(\hat{q})-u(q)-\phi d_{b}\right]=\eta \phi\left(d_{b}-d_{m}\right),
$$

where $\eta$ denotes the bargaining power of a consumer, and $1-\eta$ is the bargaining power of a producer. The trade surplus in the secondary bond market is $u(\hat{q})-u(q)-\phi d_{m}$, where $u(\hat{q})-$ $u(q)-\phi d_{b}$ is the consumer's surplus, and $\phi\left(d_{b}-d_{m}\right)$ is the producer's surplus. An active agent's

\footnotetext{
${ }^{12}$ Kalai bargaining is discussed in more detail in Aruoba et al. (2007), and Rocheteau and Wright (2005). For its application to financial markets, see Berentsen et al. (2016), Geromichalos and Herrenbrueck (2016a, b), and Huber and Kim (2016). A detailed discussion of its monotonicity properties is provided by Chun and Thomson (1988).
} 
decision problem is

$$
K\left(m_{c}, m_{p}, b_{c}, b_{p}\right) \equiv \max _{d_{m}, d_{b}}\left[u(\hat{q})-u(q)-\phi d_{m}\right] \quad \text { s.t. } \quad(12) \text { and }(13) .
$$

Note that if $K\left(m_{c}, m_{p}, b_{c}, b_{p}\right)$ is differentiable with respect to $x=m_{c}, m_{p}, b_{c}, b_{p}$, then

$$
\frac{\partial K}{\partial x}=u^{\prime}(\hat{q}) \frac{\partial \hat{q}}{\partial x}-u^{\prime}(q) \frac{\partial q}{\partial x}-\phi \frac{\partial d_{m}}{\partial x} .
$$

If the budget constraints for producers and consumers (12) are non-binding, i.e., if $\phi m_{p}>\phi d_{m}$ and $\phi b_{c}>\phi d_{b}$, then the first-order condition of the maximization problem in (14) with respect to $d_{m}$ is

$$
u^{\prime}(\hat{q}) \frac{\partial \hat{q}}{\partial d_{m}}-\phi=\phi\left[u^{\prime}(\hat{q})-1\right]=0,
$$

which means that active consumers can consume the efficient quantity, such that $u^{\prime}(\hat{q})=1$. Finally, we can derive the value function of a consumer and a producer before entering the secondary bond market as

$$
\begin{aligned}
V_{1}^{c}\left(m_{c}, b_{c}\right) & =\delta \eta K\left(m_{c}, m, b_{c}, b\right)+V_{2}^{c}\left(m_{c}, b_{c}\right), \\
V_{1}^{p}\left(m_{p}, b_{p}\right) & =\delta_{p}(1-\eta) K\left(m, m_{p}, b, b_{p}\right)+V_{2}^{p}\left(m_{p}, b_{p}\right),
\end{aligned}
$$

when the trading partner has a portfolio $(m, b)$.

\section{$5 \quad$ Monetary Equilibrium}

We focus on symmetric, stationary monetary equilibria, where all agents follow identical strategies and where real variables are constant over time. The gross growth rate of bonds is denoted by $\zeta \equiv B / B_{-1}$, and the gross growth rate of the money supply is denoted by $\gamma \equiv M / M_{-1}$. In a stationary monetary equilibrium, the real stock of money and bonds must be constant; i.e., $\phi M=\phi_{+1} M_{+1}$ and $\phi B=\phi_{+1} B_{+1}$, which implies $\gamma=\zeta=\phi / \phi_{+1}$.

In what follows, we present three stationary monetary equilibria. In the first equilibrium, labeled type-I, the producer's cash constraint and the consumer's bond constraint (12) are nonbinding in the secondary bond market. In the second equilibrium, labeled type-II, the producer's cash constraint is binding and the consumer's bond constraint is non-binding. In the third equilibrium, labeled type-III, the producer's cash constraint is non-binding and the consumer's bond constraint is binding. All proofs are relegated to the Appendix. 


\subsection{Type-I Equilibrium}

A type-I equilibrium is characterized by

$$
\begin{aligned}
u^{\prime}(\hat{q}) & =1, \\
\phi m_{p} & >\phi d_{m}, \\
\phi b_{c} & >\phi d_{b} .
\end{aligned}
$$

Equations (19) and (20) simply mean that the constraints of money and bond holdings are nonbinding in the secondary bond market.

Proposition 1 A type-I equilibrium is a list $\left\{\hat{q}, q, q_{p}, \rho\right\}$ satisfying (10) and

$$
\begin{aligned}
1 & =u^{\prime}(\hat{q}), \\
\frac{\gamma}{\beta} & =(1-n) \delta \eta\left[u^{\prime}(\hat{q})-u^{\prime}(q)\right]+(1-n) u^{\prime}(q)+n, \\
\rho & =\frac{\beta}{\gamma} .
\end{aligned}
$$

Equation (21) is obtained from the first-order condition in the secondary bond market (16) and states that active agents consume the efficient quantity; i.e., such that $u^{\prime}(\hat{q})=1$. Equation (22) is derived from the marginal value of money in the secondary bond market. The left-hand side of (22) represents the marginal cost of acquiring one additional unit of money in the primary bond market, and the right-hand side of the equation denotes the marginal benefit. With probability $1-n$, the agent will be a consumer in the goods market, in which case he has the marginal utility $u^{\prime}(q)$. With probability $(1-n) \delta$, he will be an active consumer, in which case an additional unit of money has a lower marginal benefit for him, $\eta\left[u^{\prime}(\hat{q})-u^{\prime}(q)\right]<0$, since he is already able to consume the efficient quantity. Finally, with probability $n$, the agent will be a producer in the goods market, in which case he receives a marginal utility of 1. Equation (23) is derived from the marginal value of bonds in the secondary bond market and states that bonds are priced at their fundamental value, $\beta / \gamma$, in the primary bond market.

\subsection{Type-II Equilibrium}

A type-II equilibrium is characterized by

$$
\begin{aligned}
u^{\prime}(\hat{q}) & >1 \\
\phi m_{p} & =\phi d_{m}, \\
\phi b_{c} & >\phi d_{b} .
\end{aligned}
$$

Equation (24) means that an active consumer does not consume the optimal amount of goods in the goods market, because the constraint on the producer's money holdings is binding in the secondary bond market (25). As in the type-I equilibrium, the meaning of (26) is that the constraint on the consumer's bond holdings is non-binding in the secondary bond market. 
Proposition 2 A type-II equilibrium is a list $\left\{\hat{q}, q, q_{p}, \rho\right\}$ satisfying (10) and

$$
\begin{aligned}
\hat{q} & =2 q \\
\frac{\gamma}{\beta} & =(1-n) \delta \eta\left[u^{\prime}(\hat{q})-u^{\prime}(q)\right]+(1-n) u^{\prime}(q)+n+n \delta^{p}(1-\eta)\left[u^{\prime}(\hat{q})-1\right] \\
\rho & =\frac{\beta}{\gamma} .
\end{aligned}
$$

Equation (27) is a direct consequence of the active consumers' binding cash constraint in the goods market and the fact that active producers transfer all their money to their trading partner in the secondary bond market. Equation (28) is similar to (22), except for the last term on the right-hand side of the equation. Because the cash constraint of an active producer is binding in the secondary bond market, he can earn a strictly positive surplus on his money holdings $u^{\prime}(\hat{q})-1$, according to his bargaining power $1-\eta$. As in the type-I equilibrium, bonds are priced at their fundamental value, (29), because the bond constraint of an active consumer is non-binding.

\subsection{Type-III Equilibrium}

A type-III equilibrium is characterized by

$$
\begin{aligned}
u^{\prime}(\hat{q}) & >1, \\
\phi m_{p} & >\phi d_{m}, \\
\phi b_{c} & =\phi d_{b} .
\end{aligned}
$$

Also in the type-III equilibrium, an active consumer does not consume the efficient quantity. Equation (31) means that the producer's cash constraint is non-binding in the secondary bond market, while (32) means that the consumer's bond constraint is binding in the secondary bond market.

Proposition 3 A type-III equilibrium is a list $\left\{\hat{q}, q, q_{p}, \rho\right\}$ satisfying (10) and

$$
\begin{aligned}
\mathcal{B} & =\frac{(1-\eta)[u(\hat{q})-u(q)]+\eta(\hat{q}-q)}{q}, \\
\frac{\gamma}{\beta} & =(1-n)\left[\delta u^{\prime}(\hat{q}) \frac{\eta+(1-\eta) u^{\prime}(q)}{\eta+(1-\eta) u^{\prime}(\hat{q})}+(1-\delta) u^{\prime}(q)\right]+n, \\
\rho & =\frac{\beta}{\gamma}\left[1+(1-n) \delta \eta \frac{u^{\prime}(\hat{q})-1}{\eta+(1-\eta) u^{\prime}(\hat{q})}\right] .
\end{aligned}
$$

Equation (33) is derived from the Kalai condition (13). Equation (34) is derived from the marginal value of money in the secondary bond market. With probability $(1-n) \delta$, an agent will be an active consumer, in which case he obtains a share of the surplus equal to $u^{\prime}(\hat{q})\left[\eta+(1-\eta) u^{\prime}(q)\right]\left[\eta+(1-\eta) u^{\prime}(\hat{q})\right]^{-1}$. With probability $(1-n)(1-\delta)$, he will be a passive consumer and obtain the marginal utility $u^{\prime}(q)$. With probability $n$, he will be a producer in the 
goods market, in which case he receives a marginal utility of 1 , because his cash constraint is non-binding in the secondary bond market. Equation (35) is derived from the marginal value of bonds in the secondary bond market and states that bonds exhibit a liquidity premium equal to $(1-n) \delta \eta\left[u^{\prime}(\hat{q})-1\right]\left[\eta+(1-\eta) u^{\prime}(\hat{q})\right]^{-1}$. The reason for this result is the binding bond constraint of consumers in the secondary bond market. ${ }^{13}$

\section{Regions of Existence}

Hereafter, we derive the regions of existence of each type of equilibrium with respect to the inflation rate $\gamma$ and the bond-to-money ratio $\mathcal{B}=B / M=b / \mathrm{m}$. We focus on an economy where the efficient allocation is not attainable; i.e, $\gamma>\beta$ and $\delta<1 .{ }^{14}$ Furthermore, we assume that the secondary bond market features search, $\delta \in(0,1)$, and bargaining frictions, $\eta>0$. Note that $\phi b=\phi m b / m=\mathcal{B} \phi m=\mathcal{B} q$ holds in any type of equilibrium. For now, we follow the literature that builds on Lagos and Wright (2005) and assume that the fiscal policy is purely passive. That is, we assume that any change in $\mathcal{B}$ has no effect on the inflation rate $\gamma$. We will show later what implications it has when we relax this assumption.

The following three Propositions guarantee existence of equilibria, even though they might not be unique, which is clarified in the subsequent four lemmas.

Proposition 4 There exists a constant $\gamma_{12}$ and a function $\mathcal{B}_{13}(\gamma)$ such that the type-I equilibrium is supported if, and only if, $\gamma<\gamma_{12}$ and $\mathcal{B}>\mathcal{B}_{13}(\gamma)$.

Proposition 5 There exists a function $\mathcal{B}_{23}(\gamma)$ such that the type-II equilibrium is supported if, and only if, $\gamma>\gamma_{12}$ and $\mathcal{B}>\mathcal{B}_{23}(\gamma)$.

Proposition 6 There exists a function $\mathcal{B}_{32}(\gamma)$ such that the type-III equilibrium is supported if, and only if, (i) $\gamma \leq \gamma_{12}$ and $\mathcal{B}<\mathcal{B}_{13}(\gamma)$; or (ii) $\gamma>\gamma_{12}$ and $\mathcal{B}<\mathcal{B}_{32}(\gamma)$.

The following lemma characterizes the properties of $\mathcal{B}_{13}(\gamma), \mathcal{B}_{23}(\gamma)$, and $\mathcal{B}_{32}(\gamma)$.

Lemma $7 \mathcal{B}_{13}(\gamma), \mathcal{B}_{23}(\gamma)$, and $\mathcal{B}_{32}(\gamma)$ satisfy the following properties;

(i) $\mathcal{B}_{13}(\gamma)$ is increasing in $\gamma<\gamma_{12}$,

\footnotetext{
${ }^{13}$ See Geromichalos and Herrenbrueck (2016a) for a more detailed analysis of the conditions which must hold for a liquidity premium to exist in the primary bond market.

${ }^{14}$ It is easy to show that under competitive pricing, the type-I equilibrium coincides with the type-I equilibrium under Kalai bargaining for $\eta=1$ (see Berentsen et al., 2014, 2016, and Huber and Kim, 2016). With $\delta=$ 1 and $\eta=1$, the type-I equilibrium only exists at the Friedman rule $\left(\gamma_{12}=\beta\right)$; i.e., the efficient allocation, $u^{\prime}\left(q^{*}\right)=1$, is only attainable at $\gamma=\beta$. In contrast, under Kalai bargaining, it holds that $q=\hat{q}=q^{*}$ for $\delta=1$ and $\eta<1$ for $\beta<\gamma<\operatorname{Min}\left(\gamma_{12}, \gamma_{13}\right)$, with $\gamma_{12}=\beta\left\{(1-n) \eta+(1-n)(1-\eta) u^{\prime}\left(0.5 q^{*}\right)+n\right\}$, and with $\gamma_{13}=\beta\left\{(1-n) \eta+(1-n)(1-\eta) u^{\prime}(q)+n\right\}$, where $\mathcal{B} q=(1-\eta)\left[u\left(q^{*}\right)-u(q)\right]+\eta\left(q^{*}-q\right)$ by Proposition 4 . Hence, for $\delta=1$ and $\eta<1$, the efficient allocation is attainable for inflation rates above the Friedman rule, which is shown by Geromichalos and Herrenbrueck (2016a).
} 
(ii) $\mathcal{B}_{23}(\gamma)$ is increasing in $\gamma>\gamma_{12}$,

(iii) $\mathcal{B}_{32}(\gamma)$ is increasing in $\gamma>\gamma_{12}$,

(iv) $\lim _{\gamma \rightarrow \gamma_{12}} \mathcal{B}_{13}(\gamma)=\lim _{\gamma \rightarrow \gamma_{12}} \mathcal{B}_{23}(\gamma)=\lim _{\gamma \rightarrow \gamma_{12}} \mathcal{B}_{32}(\gamma)$

The functions and critical values described in Propositions 4-6 are visualized in Figure 1 for ease of understanding. The figure shows that for $\gamma<\gamma_{12}$ only the type-I and the typeIII equilibrium exist. Which equilibrium prevails depends on the bond-to-money ratio. For $\mathcal{B}<\mathcal{B}_{13}(\gamma)$, the type-III equilibrium exists and for $\mathcal{B}>\mathcal{B}_{13}(\gamma)$, the type-I equilibrium. On the other hand, for $\gamma>\gamma_{12}$ only the type-II and the type-III equilibrium exist. If the bond supply $\mathcal{B}$ is small, the type-III equilibrium prevails, and otherwise the type-II equilibrium.

(A) $\mathcal{B}_{32}(\gamma)>\mathcal{B}_{23}(\gamma)$

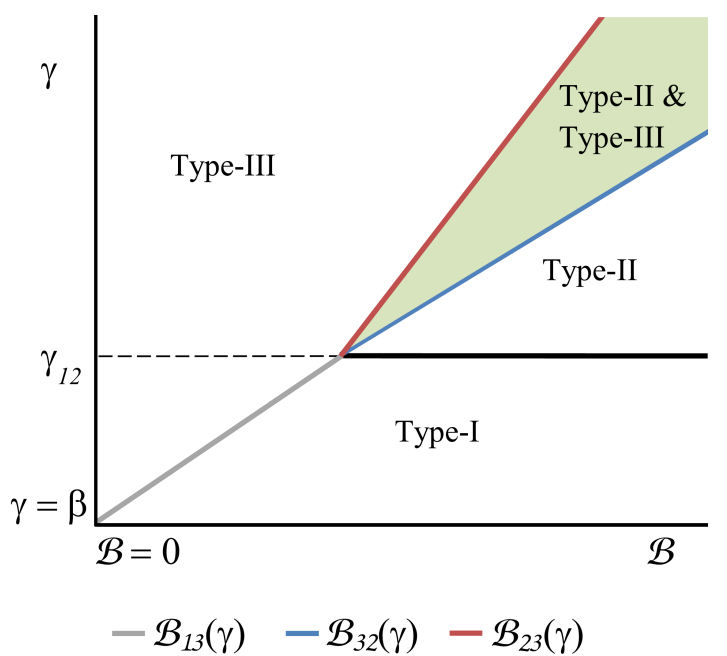

(в) $\mathcal{B}_{32}(\gamma)<\mathcal{B}_{23}(\gamma)$

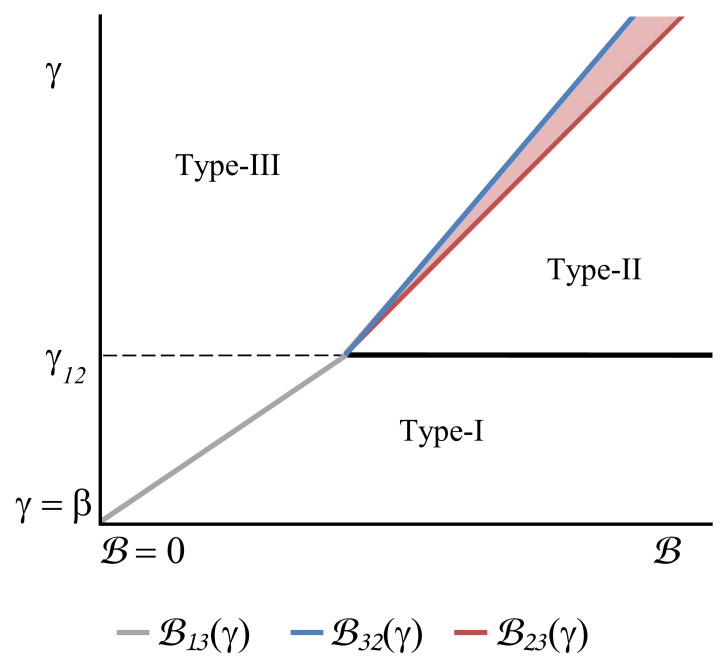

Figure 1: Regions of existence

Figure 1 shows that depending on parameter values and functional forms, there either exists a region where the type-II and the type-III equilibrium coexist for $\gamma>\gamma_{12}$ or none of the equilibria exist. Figure $1(\mathrm{~A})$ shows that when $\mathcal{B}_{32}(\gamma)>\mathcal{B}_{23}(\gamma)$ for $\gamma>\gamma_{12}$, then the region between $\mathcal{B}_{23}(\gamma)$ and $\mathcal{B}_{32}(\gamma)$ supports the type-II and the type-III equilibrium. ${ }^{15}$ Figure $1(\mathrm{~B})$ shows that when

\footnotetext{
${ }^{15}$ When $\mathcal{B}_{23}(\gamma) \leq \mathcal{B}_{32}(\gamma)$ for $\gamma>\gamma_{12}$, the region between $\mathcal{B}_{23}(\gamma)$ and $\mathcal{B}_{32}(\gamma)$ also supports an equilibrium in which the bond constraint of active consumers and the money constraint of active producers are binding simultaneously. This equilibrium is different from the type-II and the type-III equilibrium. It is a list $\left\{\hat{q}, q, q_{p}, \rho\right\}$ satisfying (10) and $\hat{q}=2 q, \mathcal{B} q=(1-\eta)[u(\hat{q})-u(q)]+\eta(\hat{q}-q), \gamma / \beta \leq(1-n) \delta \eta\left[u^{\prime}(\hat{q})-u^{\prime}(q)\right]+(1-n) u^{\prime}(q)+n+$ $n \delta^{p}(1-\eta)\left[u^{\prime}(\hat{q})-1\right], \gamma / \beta \geq(1-n)\left[\left\{\delta u^{\prime}(\hat{q})\left[\eta+(1-\eta) u^{\prime}(q)\right]\left[\eta+(1-\eta) u^{\prime}(\hat{q})\right]^{-1}+(1-\delta) u^{\prime}(q)\right\}\right]+n, \rho \geq \beta / \gamma$ $\rho \leq \beta\left\{1+(1-n) \delta \eta\left[u^{\prime}(\hat{q})-1\right]\left[\eta+(1-\eta) u^{\prime}(\hat{q})\right]^{-1}\right\} / \gamma$. The proof is available on request.
} 
$\mathcal{B}_{32}(\gamma)<\mathcal{B}_{23}(\gamma)$ for $\gamma>\gamma_{12}$, then the region between $\mathcal{B}_{32}(\gamma)$ and $\mathcal{B}_{23}(\gamma)$ does not support trading in the secondary financial market, so that active and passive agents consume the same quantity. ${ }^{16}$

The following two lemmas show the existence of an overlapping region supporting the type-II and the type-III equilibrium, which is highlighted in green in Figure 1(A).

Lemma 8 If $u^{\prime}(2 q)-\eta u^{\prime}(q)+\eta>0$ for all $q<q^{*} / 2$, then $\mathcal{B}_{32}(\gamma)>\mathcal{B}_{23}(\gamma)$ for all $\gamma>\gamma_{12}$.

Lemma 9 If there exists a constant $a<q^{*} / 2$ with $u^{\prime}\left(q^{*}\right)=1$, such that $u^{\prime}(2 q)-\eta u^{\prime}(q)+\eta>0$ for all $a<q<q^{*} / 2$, then $\mathcal{B}_{32}(\gamma)>\mathcal{B}_{23}(\gamma)$ for small $\gamma>\gamma_{12}$.

The following lemma shows the existence of a region which does not support trading in the secondary financial market for large $\gamma>\gamma_{12}$. This region is highlighted in red in Figure 1(B).

Lemma 10 If there exists a constant $a$, such that $u^{\prime}(2 q)-\eta u^{\prime}(q)+\eta<0$ for all $q<a$, then $\mathcal{B}_{32}(\gamma)<\mathcal{B}_{23}(\gamma)$ for large $\gamma>\gamma_{12}$.

Having derived the regions of existence, we are now in the position to proceed with the main finding of our paper.

\section{Optimal Quantity of Bonds}

In this section, we show how to find the optimal bond-to-money ratio, which coincides with the optimal quantity of bonds. For this purpose, we first need to derive the welfare function:

$$
(1-\beta) \mathcal{W} \equiv U\left(x^{*}\right)-x^{*}+(1-n)[\delta u(\hat{q})+(1-\delta) u(q)]-n q_{p},
$$

where $U\left(x^{*}\right)-x^{*}$ denotes the agent's utility in the primary bond market, $(1-n)[\delta u(\hat{q})+(1-\delta) u(q)]$ denotes the agent's expected utility in the goods market if he turns out to be a consumer, and $-n q_{p}$ denotes the expected utility of an agent if he becomes a producer in the goods market. Differentiating (36) with respect to $\mathcal{B}$ yields

$$
(1-\beta) \frac{\partial \mathcal{W}}{\partial \mathcal{B}}=(1-n)\left[\delta u^{\prime}(\hat{q}) \frac{\partial \hat{q}}{\partial \mathcal{B}}+(1-\delta) u^{\prime}(q) \frac{\partial q}{\partial \mathcal{B}}\right]-n \frac{\partial q_{p}}{\partial \mathcal{B}} .
$$

In the type-I and the type-II equilibrium, we always have $\partial \hat{q} / \partial \mathcal{B}=\partial q / \partial \mathcal{B}=\partial q_{p} / \partial \mathcal{B}=0$, and hence $(1-\beta) \partial \mathcal{W} / \partial \mathcal{B}=0$. In the type-III equilibrium, we also have $(1-\beta) \partial \mathcal{W} / \partial \mathcal{B}=0$ for $\delta=0$ or $\delta \eta=1 .{ }^{17}$ In contrast, for $0<\delta<1$, welfare critically depends on the bond-to-money ratio. Proposition 11 formulates a condition under which it is optimal to increase or decrease the bond-to-money ratio.

\footnotetext{
${ }^{16}$ More precisely, this equilibrium is a list $\left\{\hat{q}, q, q_{p}, \rho\right\}$ satisfying $(10)$ and $\hat{q}=q, \gamma / \beta=(1-n) u^{\prime}(q)+n, \rho=\beta / \gamma$.

${ }^{17}$ To see this, not that if $\delta=0$, then $q$ is independent of $\mathcal{B}$ by (34). Since $\delta=0, \mathcal{W}$ does not depend on $\hat{q}$, but only depends on $q$. Therefore, $\partial \mathcal{W} / \partial \mathcal{B}=0$. If $\delta \eta=1$, then $\hat{q}$ is independent of $\mathcal{B}$ by (34). Since $\delta=1, \mathcal{W}$ does not depend on $q$, but only depends on $\hat{q}$. Therefore, $\partial \mathcal{W} / \partial \mathcal{B}=0$.
} 
Proposition 11 Let $(\gamma, \mathcal{B})$ support the type-III equilibrium. (i) If $\Theta(\gamma, \mathcal{B})>0$, then welfare is increasing in $\mathcal{B}$; (ii) If $\Theta(\gamma, \mathcal{B})<0$, then welfare is decreasing in $\mathcal{B}$, where

$$
\begin{gathered}
\Theta(\gamma, \mathcal{B}) \equiv \delta\left[u^{\prime}(\hat{q}(\gamma, \mathcal{B}))-1\right] \frac{\partial \hat{q}}{\partial \mathcal{B}}+(1-\delta)\left[u^{\prime}(q(\gamma, \mathcal{B}))-1\right] \frac{\partial q}{\partial \mathcal{B}}, \\
\frac{\partial \hat{q}}{\partial \mathcal{B}}=\frac{q(\gamma, \mathcal{B})}{\eta+(1-\eta) u^{\prime}(\hat{q}(\gamma, \mathcal{B}))}+\frac{\mathcal{B}+\eta+(1-\eta) u^{\prime}(q(\gamma, \mathcal{B}))}{\eta+(1-\eta) u^{\prime}(\hat{q}(\gamma, \mathcal{B}))} \frac{\partial q}{\partial \mathcal{B}}>0, \\
\frac{\partial q}{\partial \mathcal{B}}=\frac{A(\hat{q}(\gamma, \mathcal{B}), q(\gamma, \mathcal{B}))}{B(\hat{q}(\gamma, \mathcal{B}), q(\gamma, \mathcal{B}), \mathcal{B})}<0
\end{gathered}
$$

with

$$
\begin{aligned}
A(\hat{q}, q) & \equiv-(1-n) \delta u^{\prime \prime}(\hat{q}) q\left[\eta+(1-\eta) u^{\prime}(q)\right] \eta \\
B(\hat{q}, q, \mathcal{B}) & \equiv(1-n) \delta u^{\prime \prime}(\hat{q})\left[\mathcal{B}+\eta+(1-\eta) u^{\prime}(q)\right]\left[\eta+(1-\eta) u^{\prime}(q)\right] \eta \\
& +(1-n) u^{\prime \prime}(q)\left[\eta+(1-\eta) u^{\prime}(\hat{q})\right]^{2}\left[\eta(1-\delta)+(1-\eta) u^{\prime}(\hat{q})\right] .
\end{aligned}
$$

In Proposition 11, we show that the contribution of $\partial \hat{q} / \partial \mathcal{B}$ is positive, while the contribution of $\partial q / \partial \mathcal{B}$ is negative. The reason for $\partial q / \partial \mathcal{B}$ being negative is as follows. In the secondary bond market, agents have the possibility to trade money for bonds after the realization of their idiosyncratic preference shock. The possibility to do so decreases the demand for money and hence its value. Decreasing the bond-to-money ratio helps to mitigate this externality in the type-III equilibrium and induces agents to increase their demand for money.

The following theorem states that if an economy is in the type-I equilibrium, then it is always optimal to decrease the bond-to-money ratio such that the bond constraint of active consumers becomes binding in the secondary bond market and the type-III equilibrium exists.

Theorem 12 Let $(\gamma, \mathcal{B})$ support the type-I equilibrium. Then, welfare will be improved by decreasing $\mathcal{B}$.

The reasoning behind the result stated in Theorem 12 is as follows. In the type-I equilibrium, since $\gamma<\gamma_{12}$ and $\mathcal{B}>\mathcal{B}_{13}(\gamma)$, a strategy that decreases the bond-to-money ratio will not affect welfare. However, at $\mathcal{B}=\mathcal{B}_{13}(\gamma)$, welfare will be further improved by decreasing $\mathcal{B}$. For ease of understanding, Figure 2 stylistically shows the evolution of welfare as a function of $\mathcal{B}$ for $\gamma<\gamma_{12}$ and intermediate values of $\delta$ and $\eta$. The figure shows that for $\eta<1$, the welfare-optimal bondto-money ratio, $\mathcal{B}^{*}$, is in the range $0<\mathcal{B}^{*}<\mathcal{B}_{13}(\gamma)$. Furthermore, welfare at $\mathcal{B}=0$ is lower than at $\mathcal{B}_{13}(\gamma)$ if $\eta$ is low enough.

For $\eta=1$, one can show that prices and quantities characterized by Proposition 1 and 3 coincide with competitive pricing in the secondary bond market. ${ }^{18}$ From Proposition 11, for $\eta=1$ and $0<\delta<1$, one can also show that welfare is uniquely maximized at $\mathcal{B}^{*}=0$, if $\gamma \leq \gamma_{12}$

\footnotetext{
${ }^{18}$ For a formal proof of this argument, we refer the interested reader to Huber and Kim (2016).
} 


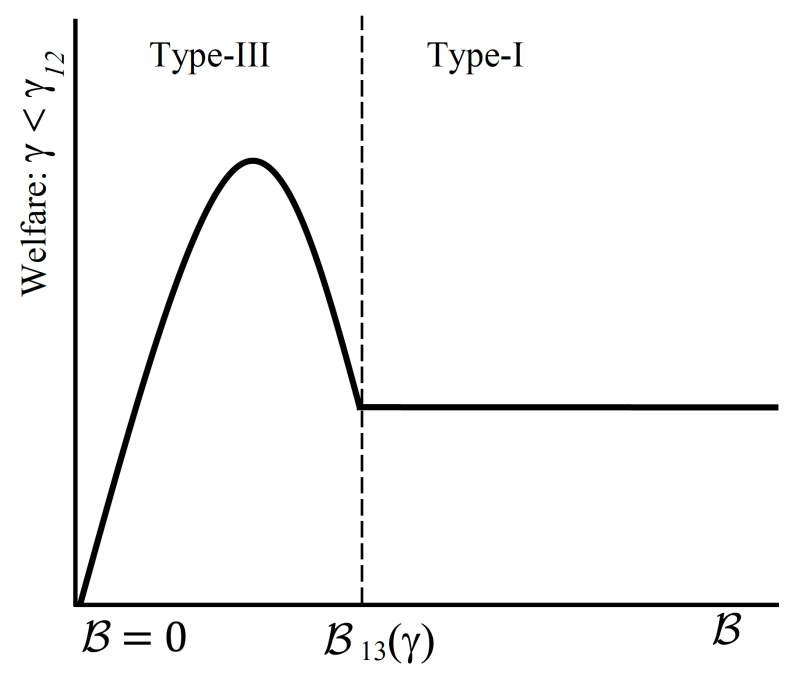

Figure 2: Welfare as a FunCtion of $\mathcal{B}$ FOR $\gamma<\gamma_{12}$

and $\left[u^{\prime \prime}(q)\right]^{2}>\left[u^{\prime}(q)-1\right] u^{\prime \prime \prime}(q)$ for all $q \in\left(q^{*} / 2, q^{*}\right] .{ }^{19}$ Note, however, that for a fully competitive secondary bond market without search frictions, i.e., $\eta=1$ and $\delta=1$, our results cease to hold. To see this, note that for $\eta=1$ and $\delta=1$, the solution of $\hat{q}$ is completely determined by equation (34); i.e., $\hat{q}$ is not a function of $\mathcal{B}$. Since every agent is active with $\delta=1$, welfare is determined by the consumption of active agents, $\hat{q}$, which is independent of $\mathcal{B}$. Hence, search frictions are a key ingredient for our results to persist. ${ }^{20}$

For inflation rates above $\gamma_{12}$, either the type-II or the type-III equilibrium exists. Since welfare is continuous in each type of equilibrium and since welfare is maximized (in terms of $\mathcal{B}$ ) in the interior of the region of the type-III equilibrium for $\gamma=\gamma_{12}$, then it must also be maximized in the interior of the region of the type-III equilibrium, when $\gamma>\gamma_{12}$ and $\gamma$ is sufficiently close to $\gamma_{12}$ by continuity. Hence, only when $\gamma$ is considerably higher than $\gamma_{12}$, is it not optimal to reduce $\mathcal{B}$ in the type-II equilibrium. That is, $\mathcal{B}^{*} \geq \mathcal{B}_{23}(\gamma)$. This result is shown graphically in Figure 3 for intermediate values of $\delta$ and $\eta$ with $u^{\prime}(2 q)-\eta u^{\prime}(q)+\eta>0$ and consequently $\mathcal{B}_{32}(\gamma)>\mathcal{B}_{23}(\gamma)$ for all $\gamma>\gamma_{12}$.

Figure 3 shows that for large $\gamma>\gamma_{12}$ welfare is maximized in the type-II equilibrium, and the optimal policy is to keep the bond-to-money ratio at least at the border of the type-II equilibrium. The figure also shows that $\mathcal{B}^{*}$ is not continuous between the type-II and the type-III equilibrium,

\footnotetext{
${ }^{19}$ The proof is available on request. For $\eta=1$ and $0<\delta<1$, the condition $\left[u^{\prime \prime}(q)\right]^{2}>\left[u^{\prime}(q)-1\right] u^{\prime \prime \prime}(q)$ for all $q \in\left(q^{*} / 2, q^{*}\right]$ is satisfied for a wide range of parameter values and functional forms of the utility function. Note, however, that for some large $\gamma>\gamma_{12}$ we have $\left[u^{\prime \prime}(q)\right]^{2}<\left[u^{\prime}(q)-1\right] u^{\prime \prime \prime}(q)$ for all $q \leq \hat{q}\left(\gamma, \mathcal{B}_{32}(\gamma)\right)$, which results in $\Theta(\gamma, 0)=0$ and $\Theta(\gamma, \mathcal{B})>0$ for $\mathcal{B} \in\left(0, \mathcal{B}_{32}(\gamma)\right]$, such that welfare is consequently minimized at $\mathcal{B}=0$.

${ }^{20}$ The result that welfare is declining in the supply of the illiquid asset for a competitive secondary bond market with partial access $(\eta=1$ and $0<\delta<1)$ and $\gamma<\gamma_{12}$ is also confirmed by Geromichalos and Herrenbrueck (2017).
} 




Figure 3: Regions of EXistence And optimal $\mathcal{B}$

hence there is a jump in $\mathcal{B}^{*}$ for high inflation rates, which is shown by the following proposition.

Proposition 13 Let $u^{\prime}(2 q)-\eta u^{\prime}(q)+\eta>0$ for $q<q^{*} / 2$. Given $\gamma>\gamma_{12}$, for any $\mathcal{B} \in$ $\left(\mathcal{B}_{23}(\gamma), \mathcal{B}_{32}(\gamma)\right)$, welfare is higher under the type-II equilibrium than under the type-III equilibrium.

The reason behind this result is that the consumed quantity of active and passive consumers in the type-II equilibrium is higher than in the type-III equilibrium for any $\mathcal{B} \in\left(\mathcal{B}_{23}(\gamma), \mathcal{B}_{32}(\gamma)\right)$. Therefore, for small values of $\varepsilon$, any $\mathcal{B} \in\left(\mathcal{B}_{23}(\gamma)-\varepsilon, \mathcal{B}_{23}(\gamma)\right)$ cannot be the optimal value of $\mathcal{B}$ by continuity, because welfare under the type-II equilibrium with $\mathcal{B}=\mathcal{B}_{23}(\gamma)$ would be higher.

\section{Discussion}

In this section, we discuss the evolution of the secondary bond market price of bonds, the assumption of purely passive fiscal policy, the empirical evidence of market frictions, the Friedman rule, and the optimal short-term interest rate.

The Price of Bonds in the Secondary Bond Market In our model, bonds are issued in a competitive market and are subsequently traded in a secondary bond market. Hereafter, we have a closer look at the evolution of the secondary bond market price of bonds, over which agents bargain in bilateral meetings. In particular, the secondary bond market price of bonds, labeled as $\varphi$ hereafter, is given by the trading amount of money that active producers offer, $\phi d_{m}$, 
divided by the trading amount of bonds that active consumers want to sell, $\phi d_{b}$. Using the Kalai constraint (13) and $\phi d_{m}=\hat{q}-q$, we find that in each equilibrium it holds that

$$
\varphi=\left[(1-\eta) \frac{u(\hat{q})-u(q)}{\hat{q}-q}+\eta\right]^{-1} .
$$

From the previous section, we already know that in the type-I and in the type-II equilibrium we have $\partial \hat{q} / \partial \mathcal{B}=\partial q / \partial \mathcal{B}=0$, and therefore also $\partial \varphi / \partial \mathcal{B}=\partial \rho / \partial \mathcal{B}=0$. Furthermore, from Proposition 11, we have $\partial \hat{q} / \partial \mathcal{B}>0$ and $\partial q / \partial \mathcal{B}<0$ in the type-III equilibrium. Thus, it is easy to show that $\partial \rho / \partial \mathcal{B}<0$. In the type-III equilibrium, the sign of $\partial \varphi / \partial \mathcal{B}$ depends on whether $\partial \hat{q} / \partial \mathcal{B}>0$ or $\partial q / \partial \mathcal{B}<0$ dominates. We find that for large values of $\eta$ and $\delta$, we have $\partial \varphi / \partial \mathcal{B}<0$, while for low values of $\eta$ and $\delta$, we have $\partial \varphi / \partial \mathcal{B}>0$. These findings are formalized in the following proposition.

Proposition 14 Let $(\gamma, \mathcal{B})$ support the type-III equilibrium. Then, we always have $\partial \rho / \partial \mathcal{B}<0$. If the values of $\delta$ and $\eta$ are large, we have $\partial \varphi / \partial \mathcal{B}<0$, and if the values of $\delta$ and $\eta$ are small, we have $\partial \varphi / \partial \mathcal{B}>0$.

Thus, for large values of $\delta$ and $\eta$ the price of short-dated bonds agreed on in the secondary bond market is likely to move in the same direction as the price of bonds in the primary bond market after a reduction in the bond supply; i.e., $\partial \varphi / \partial \mathcal{B}<0$. However, our results indicate that for secondary bond markets that feature severe search and bargaining frictions, the opposite may occur.

Passive Fiscal Policy Up to now, we assumed that any change in $\mathcal{B}$ has no effect on the inflation rate $\gamma$. We showed that under this assumption it can be optimal to reduce $\mathcal{B}$ in order to improve welfare. However, one can argue that it is easier for the central bank to determine the lump-sum transfer $\tau$ and that it does not explicitly control $\gamma$. From the central bank's budget constraint (1), we have

$$
\gamma-1-\tau=\mathcal{B}(1-\rho \gamma)
$$

In the type-III equilibrium, we have $\partial \rho / \partial \mathcal{B}<0$ and hence any reduction in $\mathcal{B}$ results in an increase in $\rho$. Thus, reducing $\mathcal{B}$ results in a reduction in the value of the right-hand side of the above equation and the equality can only be satisfied if $\gamma$ declines. Moreover, welfare is decreasing with respect to $\gamma$ by the following proposition.

Proposition 15 In any type of equilibrium, it holds that $\partial q / \partial \gamma<0$. In the type-II and the type-III equilibrium, it holds that $\partial \hat{q} / \partial \gamma<0$.

Thus, assuming that $\tau$ is constant results in a reduction in the inflation rate $\gamma$ when $\mathcal{B}$ is decreased, which further improves welfare, since $\partial \hat{q} / \partial \gamma<0$ and $\partial q / \partial \gamma<0$. 
Empirical Evidence of Market Frictions Search frictions indicate how hard it is to find a trading partner in the secondary bond market. As discussed in detail in Duffie et al. (2005), search frictions are rather low in the market for U.S. Treasuries and T-Bills. ${ }^{21}$ Concretely, these frictions are mainly caused by time delays in calling a suitable counterparty. Note, however, that although the market for U.S. government bonds is among the most liquid over-the-counter markets in the world, the probability of finding a suitable counterparty is smaller than 1 for many securities according to the methodology presented in Pontrandolfo (2015). This finding indicates that the empirical value of $\delta$ is likely to be large, but smaller than 1 .

Bargaining frictions determine how the total trade surplus in the secondary bond market is split among trading partners. In a recent study, Lagos and Zhang (2015) argue that most of the trade surplus is exploited by financial intermediaries and not split among the trading partners themselves. In our model, however, we abstract from the role of financial intermediaries and are thus interested in the surplus-sharing in direct buyer-seller matching platforms. Recently, several financial institutions launched such platforms, such as UBS Bond Port, which allows trading partners to agree bilaterally on the price, without any need for a financial intermediary. ${ }^{22}$ UBS Bond Port grew quickly and reached in 2015 a yearly trading volume of around USD 32 billion, with daily access to more than 10,000 bonds. On average, more than 1,200 trades are executed on a daily basis, which allows us to obtain a good overview of the behavior of trading partners. Based on the insights of UBS, trading partners tend to agree on the mid-price, which indicates that $\eta$ is close to $0.5 .^{23}$

The Friedman Rule From Proposition 15 we know that the consumption of active and passive agents is decreasing in the inflation rate, such that the Friedman rule $(\gamma=\beta)$ achieves the first-best allocation, $q=\hat{q}=q^{*}$. At the Friedman rule, agents are able to perfectly self-insure themselves against consumption shocks, such that they do not have any desire to participate in the secondary bond market. Consequently, for $\gamma=\beta$ it is not welfare-improving to contract the bond supply.

Andolfatto (2010) and Gomis-Porqueras and Sanches (2013) propose an alternative way to increase the real return of the medium of exchange by paying interest on money. Implementing the Friedman rule or paying interest on money are both policies which increase the real value of money; however, both methods require some kind of taxation to be implemented.

\footnotetext{
${ }^{21}$ Also see Krishnamurthy (2002) for the empirical price effects of search frictions in the market for U.S. Treasuries.

${ }^{22}$ Other providers are, for instance, Liquidnet Fixed Income and HSBC Credit Place.

${ }^{23}$ An estimate of $\eta=0.5$ is also in line with insights from experimental economics. For instance, Forsythe et al. (1994) conducted ultimatum (take-it-or-leave-it) games in the United States in 1988. They found that if subjects get paid, they tend to act "fairly" and share the pie evenly, which contradicts the subgame perfect Nash equilibrium (which implies an offer of 0). Roth et al. (1991) conducted ultimatum games in Israel, Japan, the United States, and Yugoslavia in 1989 and 1990. They found that the proposals made by bargainers are the highest in the United States and Yugoslavia with a modal proposal of 50 percent of the pie and the lowest in Japan and Israel with a modal proposal of 40 percent of the pie. Slonim and Roth (1998) conducted an ultimatum game in the Slovak Republic in 1994. They found that subjects offer between 41.5 percent and 44 percent and that a bigger size of the pie results in lower offers when players have the possibility to gain experience.
} 
Taxation is not required to control the bond-to-money ratio: On the contrary, governments use this ratio as a conventional policy tool. In this paper, we do not focus on the Friedman rule and explicitly analyze an economy where the efficient allocation is not attainable.

Optimal short-term Interest Rate Our results about the optimal quantity of bonds can directly be linked to the optimal short-term interest rate, which is defined as $i \equiv 1 / \rho-1$. For a given $\gamma<\gamma_{12}$, welfare is maximized in the type-III equilibrium, and hence it is optimal for bonds to be priced above their fundamental value. How large the optimal short-term interest rate will be depends on market frictions. For $\eta=1$ and $0<\delta<1$, we find that $i$ is close to zero, while it is higher for lower values of $\eta$. For large $\gamma>\gamma_{12}$, it is generally not optimal to reduce $\mathcal{B}$ from the unconstrained level, and consequently the optimal short-term interest rate equals the Fisher equation, $\gamma / \beta-1$. Hence, the optimal short-term interest rate is increasing in $\gamma$ from its initial level to the Fisher equation.

\section{$9 \quad$ Numerical Analysis}

In the previous sections, we have shown that it can be welfare-improving to reduce the bond-tomoney ratio. The optimal bond supply, $\mathcal{B}^{*}$, depends on preferences and technology. Hereafter, we calibrate the model to U.S. data to analyze the sensitivity of the optimal bond-to-money ratio $\mathcal{B}^{*}$ with respect to the inflation rate $(\gamma)$, and the market frictions $(\delta$ and $\eta)$.

We set a model period to one year and choose the following functional forms for preferences and technology: $u(q)=q^{1-\alpha} /(1-\alpha), c(q)=q$, and $U(x)=A \log (x)$. From Lemma 8 it follows that for $u(q)=q^{1-\alpha} /(1-\alpha)$, we need $\alpha \in(0,1)$ and $\eta \leq 2^{-\alpha}$ to have an overlapping region supporting the type-II and the type-III equilibrium for all $\gamma>\gamma_{12} \cdot{ }^{24}$

The following parameters need to be identified: (i) preference parameters: $(\beta, A, \alpha)$; (ii) technology parameters: $(\xi, n)$; (iii) market frictions: $(\delta, \eta)$; (iv) and policy parameters $(\mathcal{B}, \gamma)$.

The parameters are identified by using U.S. data from the first quarter of 1992 to the fourth quarter of $2016 .^{25}$ The calibration targets are shown in Table 1.

The parameters $\gamma$, and $\mathcal{B}$ can be set equal to their target values. That is, we set $\gamma=1.023$ to replicate the average annual change in the inflation rate. We interpret the bond-to-money ratio as the ratio of the outstanding T-Bills to M1, and obtain an average value of $\mathcal{B}=0.688$.

The market frictions $\delta$ and $\eta$ are determined according to the findings of the discussion section. The bargaining power of consumers in the secondary bond market is obtained from insights of direct buyer-seller matching platforms; i.e., we use a value of $\eta=0.5$. In the secondary bond market, we use the following matching function: $\xi \mathcal{M}[n,(1-n)]=\xi n(1-n)$. We follow the literature and set $n=0.5$ in order to maximize the number of matches. The matching efficiency satisfies $\xi \in(0,2)$, such that the matching probabilities of consumers and producers in the

\footnotetext{
${ }^{24}$ Note, that for $u(q)=q^{1-\alpha} /(1-\alpha), \alpha \in(0,1)$, and $\eta \leq 2^{-\alpha}$, we have $u^{\prime}(2 q)-\eta u^{\prime}(q)+\eta=2^{-\alpha} q^{-\alpha}-\eta q^{-\alpha}+\eta>0$. Hence, $\mathcal{B}_{32}(\gamma)>\mathcal{B}_{23}(\gamma)$ for all $\gamma>\gamma_{12}$.

${ }^{25}$ All data sources are provided in the Appendix. We choose this sample period, because we could not find any data on the outstanding T-Bills before 1992 .
} 
TABle 1: Calibration targets

\begin{tabular}{ll}
\hline \hline Target Description & Target Value \\
\hline Average annual price of T-Bills & 0.975 \\
Average annual inflation rate & 0.023 \\
Average bond-to-money ratio & 0.688 \\
Average money demand & 0.128 \\
Average elasticity of money demand & $-0.04(0.009)$ \\
\hline
\end{tabular}

Standard errors in parentheses.

secondary financial market equal $\delta=\delta^{p}=\xi / 2 \in(0,1)$. To obtain the baseline value of $\delta$, we consider evidence of search frictions in the market for U.S. government bonds and accordingly use a large value of $\delta=0.8 .^{26}$

The preference parameters $\beta, A$ and $\alpha$ are calibrated simultaneously by matching the average level of money demand, denoted as $\mathcal{M D}=0.128$, its elasticity with respect to the 3 -month $\mathrm{T}$ Bill rate, denoted as $\xi=-0.04$, and the average price of T-Bills, $\rho=1 /(1+i)=0.975$, where $i=0.0253$ represents the average annual 3 -month T-Bill rate. ${ }^{27}$

The model's money demand is

$$
\mathcal{M D}=\frac{\phi M_{-1}}{Y}=\frac{\phi m}{A+(1-n)[\delta \phi \hat{m}+(1-\delta) \phi m]},
$$

where $\phi m=\phi M_{-1}=q$ and $\phi \hat{m}=\hat{q} .{ }^{28}$ The model's money demand depends on the interest rate in the primary bond market $i \equiv 1 / \rho-1$, and on $\alpha$ and $A$ via $u(q)$ and $U(x)$. We estimate the model's elasticity of money demand with respect to $i$ in the same way as its empirical counterpart; i.e., by ordinary least squares and a log-log specification. Table 2 presents the calibrated parameter values, where the model replicates both money demand targets and the target price of T-Bills exactly.

The calibrated parameter values allow us to obtain the critical values $\gamma_{12}$ and $\mathcal{B}_{13}(\gamma)$, and to study the effect of a change in $\mathcal{B}$ on consumption and asset prices. In particular, we calculate the optimal value of the bond-to-money ratio, $\mathcal{B}^{*}$, which allows us to calculate the percentage of total consumption that agents would be willing to give up in order to be in a steady state with $\mathcal{B}=\mathcal{B}^{*}$ instead of $\mathcal{B}=0.688$, which is denoted as $1-\Delta$. Furthermore, we calculate the impact of this change in the bond-to-money ratio on the interest rate of bonds in the primary bond

\footnotetext{
${ }^{26}$ The baseline estimates of $\delta$ and $\eta$ are in line with Berentsen et al. (2016).

${ }^{27}$ Money demand is measured as the ratio of M1 divided by the nominal gross domestic product. The elasticity of money demand with respect to the 3-month T-Bill rate is estimated by ordinary least squares and a log-log specification. Note that since we do not know ex-ante in which equilibrium the economy is, we cannot assume that the Fisher equation holds in order to estimate $\beta$.

${ }^{28}$ The model's money demand is defined as the ratio of the real stock of money, $\phi M_{-1}=\phi m=q$, divided by the real output of the economy, $Y$. The latter term is obtained by the sum of the real output of the primary bond market, which equals $A$ for $U(x)=A \log (x)$, and the real output of the goods market, which equals $(1-n)[\delta \phi \hat{m}+(1-\delta) \phi m]$.
} 
TABle 2: CAlibrated PARAmeter values

\begin{tabular}{cccccccc}
\hline \hline$\beta$ & $\gamma$ & $\mathcal{B}$ & $\delta$ & $\eta$ & $n$ & $A$ & $\alpha$ \\
\hline 0.998 & 1.023 & 0.688 & 0.8 & 0.5 & 0.5 & 6.56 & 0.74 \\
\hline
\end{tabular}

${ }^{\dagger}$ Table 2 displays the calibrated parameter values. The parameters $\gamma, \mathcal{B}, \eta, \delta$, and $n$ are set equal to their target values, while $\beta, A$ and $\alpha$ are obtained simultaneously by matching the average money demand, its elasticity with respect to the 3-month T-Bill rate, and the average price of T-Bills.

Table 3: Calibration Results

\begin{tabular}{cccccc}
\hline \hline$\gamma_{12}$ & $\mathcal{B}_{13}(\gamma)$ & $\mathcal{B}^{*}$ & $1-\Delta$ & $\Delta_{i}$ & $s_{G M}$ \\
\hline 1.198 & 0.12 & 0.09 & 0.00001 & -0.0023 & 0.07 \\
\hline
\end{tabular}

${ }^{\ddagger}$ Table 3 displays the calibration results. The term $\gamma_{12}$ denotes the critical inflation rate that separates the type-I from the type-II equilibrium, and the term $\mathcal{B}_{13}(\gamma)$ denotes the critical bond-to-money ratio that separates the type-I from the type-III equilibrium at the calibrated value of $\gamma=1.023$. The table also shows the optimal bond-to-money ratio, $\mathcal{B}^{*}$, which is calculated such that $\Theta\left(\gamma, \mathcal{B}^{*}\right)=0$. It also shows the percentage of total consumption that agents would be willing to give up in order to be in a steady state with $\mathcal{B}=\mathcal{B}^{*}$, instead of $\mathcal{B}=0.688$, denoted as $1-\Delta$, and the effect on $i$, denoted as $\Delta_{i}$. Finally, it also shows the size of the goods market, $s_{G M}$.

market, denoted as $\Delta_{i}=1 / \rho\left(\mathcal{B}^{*}\right)-1 / \rho(\mathcal{B})$. Finally, we also provide the share of goods market consumption on total consumption, labeled as $s_{G M}$. Table 3 gives a brief overview of our results.

Table 3 shows that $\gamma_{12}=1.198>1.023$ and $\mathcal{B}_{13}(\gamma)=0.12<0.688$; i.e., that the U.S. economy is in the type-I equilibrium at the calibrated value of $\gamma$ and that T-Bills are consequently priced at their fundamental value. Furthermore, we have $\mathcal{B}_{32}(\gamma)>\mathcal{B}_{23}(\gamma)$, since $\eta=0.5<2^{-\alpha}=0.60$, and thus there exists an overlapping region supporting the type-II and the type-III equilibrium for all $\gamma>\gamma_{12}$. We find that $\mathcal{B}^{*}=0.09$, and that the benefits associated with a policy of reducing $\mathcal{B}$ to $\mathcal{B}^{*}$ are relatively low and in the region of 0.001 percent of total consumption. However, reducing $\mathcal{B}$ to $\mathcal{B}^{*}$ has a larger impact on the yield of bonds in the primary bond market; i.e., it reduces $i \equiv 1 / \rho-1$ by 0.23 percent. That is, the optimal interest rate on T-Bills equals $i+\Delta_{i}=0.023$. Finally, the share of goods market consumption on total consumption is, with 7 percent, in line with related studies.

The Role of Search and Bargaining Frictions We now investigate how sensitive our results are with respect to the search and bargaining frictions in the secondary bond market. In order to do this, we keep all parameters at their calibrated values and change $\delta$ and $\eta$ from 0 to 1 . For each value of $\delta$ and $\eta$, we then calculate whether it is optimal to reduce the bond-to-money ratio from $\mathcal{B}_{13}(\gamma)$, respectively $\mathcal{B}_{23}(\gamma)$; i.e., we calculate $\mathcal{B}_{13}(\gamma)-\mathcal{B}^{*}$ for $\gamma<\gamma_{12}$ and $\mathcal{B}_{23}(\gamma)-\mathcal{B}^{*}$ for $\gamma>\gamma_{12}$. Figure $4(\mathrm{~A})$ shows our findings for the calibrated value of $\gamma=1.023 .^{29}$

For large values of $\delta$ and $\eta$, we find that $\gamma_{12}<\gamma=1.023$, which is shown by the upper

\footnotetext{
${ }^{29}$ Remember that all critical values: $\gamma_{12}, \mathcal{B}_{13}(\gamma), \mathcal{B}_{23}(\gamma)$, and $\mathcal{B}_{32}(\gamma)$ are sensitive to changes in $\delta$ and $\eta$.
} 
(A) $\gamma=1.023$

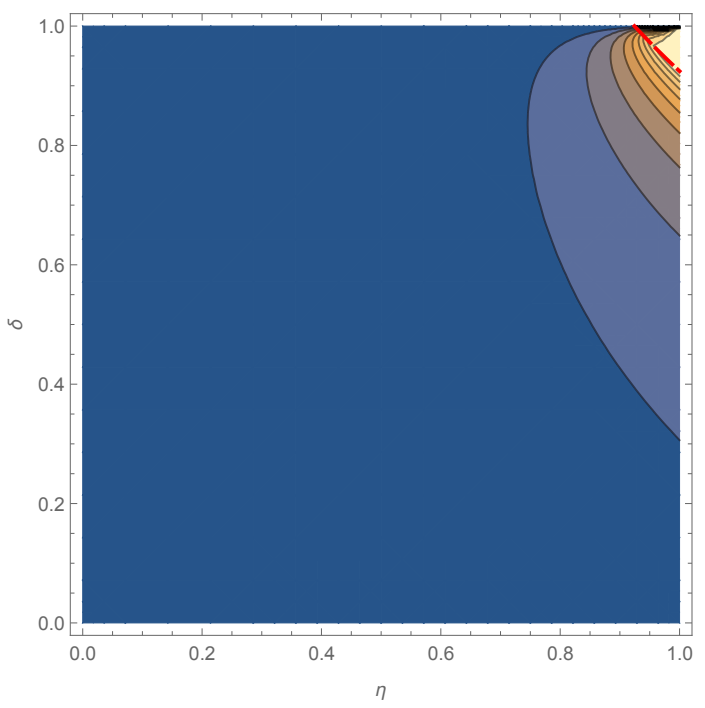

(B) $\gamma=1.198$

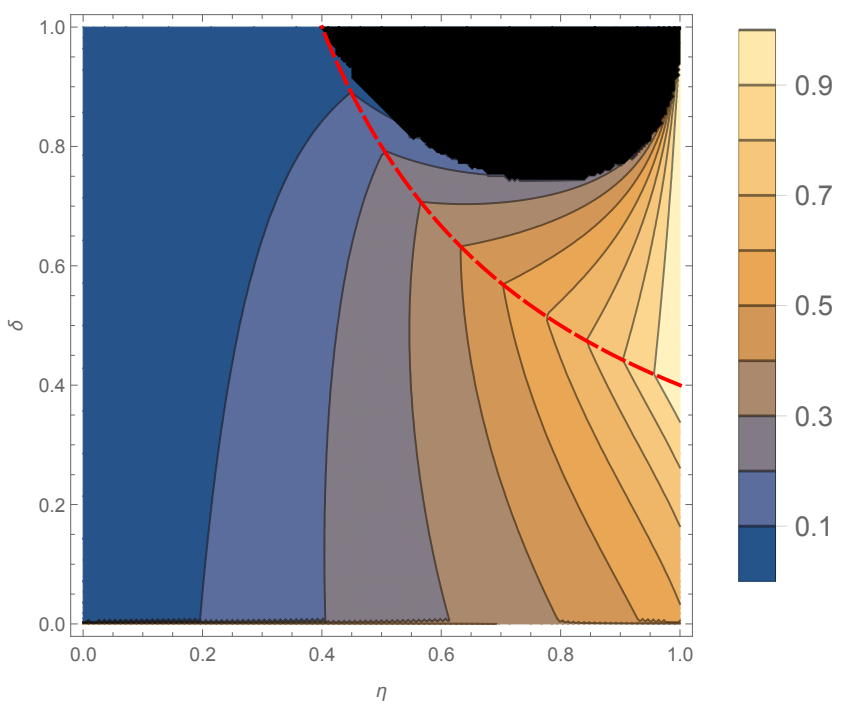

Figure 4: Optimal Reduction in $\mathcal{B}$

right region from the dashed line; i.e., in the top-right corner, the economy would be in the type-II equilibrium or the type-III equilibrium depending on $\mathcal{B}$. If $\delta$ is very high, then welfare is maximized at the type-II equilibrium, so $\mathcal{B}_{23}(\gamma)-\mathcal{B}^{*}=0$. For ease of reference, this area is shaded in black in the top-right corner. Otherwise, it is optimal to reduce $\mathcal{B}$ from the unconstrained level $\mathcal{B}_{23}(\gamma)$. In this case, we obtain the largest optimal reduction in $\mathcal{B}$ for high values of $\delta$ and $\eta$. This is because for low trading frictions bonds become more attractive and so the required reduction in $\mathcal{B}$ is large.

For the lower left region from the dashed line, $\gamma_{12}$ is high, so $\gamma_{12}>\gamma=1.023$. Hence, the economy would be in the type-I equilibrium or the type-III equilibrium depending on $\mathcal{B}$. In this region we have $\mathcal{B}_{13}(\gamma)-\mathcal{B}^{*}>0$ for any value of $\delta$ and $\eta$ (except for the corner solutions), which is in line with our theoretical finding in Theorem 12. For high values of $\eta$, the optimal reduction in $\mathcal{B}$ from $\mathcal{B}_{13}(\gamma)$ is large, independent of the value of $\delta$. This is because for high values of $\eta$ active consumers obtain a larger fraction of the trade surplus in the secondary bond market, which is why the demand for money decreases. Consequently, the optimal reduction in $\mathcal{B}$ needs to be large to correct this misallocation.

The Role of Inflation We now analyze how larger inflation rates affect our results. To this, we follow the same procedure as before; i.e., we keep all parameters at their calibrated values and change $\delta$ and $\eta$ from 0 to 1 . However, we now do these calculations for a larger gross inflation rate. In Figure 4(в), we show the results for a high inflation rate of $\gamma=1.198$, which corresponds to $\gamma_{12}$ for the calibrated values of $\eta=0.5$ and $\delta=0.8$.

Obviously, the region where $\gamma>\gamma_{12}$, which is the upper right area from the dashed line, is 
larger with a higher inflation rate of $\gamma=1.198$. In addition, the larger black area shows that the region of $\mathcal{B}_{23}(\gamma)-\mathcal{B}^{*}=0$ is increasing in the inflation rate. However, we can see that it is still optimal to reduce the bond-to-money ratio for a wide range of parameter values when $\gamma>\gamma_{12}$.

For the region $\gamma<\gamma_{12}$, which is the lower left area from the dashed line, we find that the optimal reduction in $\mathcal{B}$ is larger than at the calibrated value of $\gamma=1.023$. This is because for higher inflation rates of $\gamma<\gamma_{12}$, the demand for money is lower due to the higher opportunity cost of holding money. Therefore, the optimal reduction in the bond-to-money ratio, $\mathcal{B}_{13}(\gamma)-$ $\mathcal{B}^{*}>0$, needs to be larger in order to incentivize agents to improve their self-insurance against idiosyncratic liquidity shocks.

\section{Conclusion}

We develop a general equilibrium model, where agents can trade money for government bonds in a secondary financial market which features search and bargaining frictions. The possibility to do so reduces the incentive to self-insure against liquidity shocks, and as a consequence agents rely on other market participants to provide liquidity. In such an environment, contracting the bond supply can be welfare-improving for low inflation rates. The reason is that a lower supply makes bonds scarce and less attractive, which induces agents to increase their demand for money. We also show that this effect fails at higher inflation rates, because agents hold so little money in the first place that manipulating the bond supply is not enough to correct the misallocation.

Furthermore, we show that the optimal contraction in the bond supply from the unconstrained level depends critically on the structure of the secondary bond market and inflation. For low inflation rates and a secondary bond market which features intermediate to large trading frictions, we have $\gamma<\gamma_{12}$. In such a case, we find numerically that the optimal contraction in the bond supply is generally decreasing in bargaining frictions (increasing in $\eta$ ). This is because for higher bargaining frictions, it is less attractive to hold bonds for the purpose of selling them in a secondary market in order to benefit from liquidity held by others. Consequently, the demand for money is already high, and a small reduction in the bond supply from the unconstrained level is sufficient to correct the misallocation. For higher inflation rates and/or a secondary bond market which features low trading frictions (high values of $\delta$ and $\eta$ ), we have $\gamma>\gamma_{12}$. In such a case, choosing the optimal bond supply is more difficult. This is because under certain circumstances welfare is higher if bonds are not scarce. Therefore, reducing the bond supply might be welfare-decreasing in a such an environment. 


\section{A Appendix - For Online Publication}

\section{A.1 Proofs}

Proof of Proposition 1. Derivation of (21). Since the short-selling constraints (12) are not binding in a type-I equilibrium, it is obvious from (16) that (21) holds.

Derivation of (22). The marginal value of money of an agent at the beginning of a period can be written as follows:

$$
\frac{\partial V_{1}}{\partial m}=(1-n) \frac{\partial V_{1}^{c}}{\partial m}+n \frac{\partial V_{1}^{p}}{\partial m} .
$$

Using (17), we can rewrite the above equation as

$$
\frac{\partial V_{1}}{\partial m}=(1-n)\left[\delta \eta \frac{\partial K}{\partial m_{c}}+\frac{\partial V_{2}^{c}}{\partial m_{c}}\right]+n\left[\delta_{p}(1-\eta) \frac{\partial K}{\partial m_{p}}+\frac{\partial V_{2}^{p}}{\partial m_{p}}\right]
$$

where

$$
\begin{aligned}
\frac{\partial K}{\partial m_{c}} & :=\left.\frac{\partial K\left(m_{c}, m_{p}, b_{c}, b_{p}\right)}{\partial m_{c}}\right|_{\left(m_{c}, m_{p}, b_{c}, b_{p}\right)=(m, m, b, b)}, \\
\frac{\partial K}{\partial m_{p}} & :=\left.\frac{\partial K\left(m_{c}, m_{p}, b_{c}, b_{p}\right)}{\partial m_{p}}\right|_{\left(m_{c}, m_{p}, b_{c}, b_{p}\right)=(m, m, b, b)} .
\end{aligned}
$$

We can use the envelope condition of a consumer in the goods market, (8), to replace $\partial V_{2}^{c} / \partial m_{c}$, and (11) to replace $\partial V_{2}^{p} / \partial m_{p}$. Furthermore, in a symmetric equilibrium, we have $\partial K / \partial m_{c}=$ $\phi-\phi u^{\prime}(q)$ and $\partial K / \partial m_{p}=0$, because $\partial \hat{q} / \partial m_{c}=0, \partial q / \partial m_{c}=1 / p=\phi, \partial d_{m} / \partial m_{c}=-1$, and $\partial \hat{q} / \partial m_{p}=\partial q / \partial m_{p}=\partial d_{m} / \partial m_{p}=0$ with (15). Using these expressions to replace $\partial K / \partial m_{c}$ and $\partial K / \partial m_{p}$ and (5) updated one period, we obtain (22).

Derivation of (23). Following the same procedure as in the derivation of (22), we can rewrite the marginal value of bonds at the beginning of a period as

$$
\frac{\partial V_{1}}{\partial b}=(1-n)\left[\delta \eta \frac{\partial K}{\partial b_{c}}+\frac{\partial V_{2}^{c}}{\partial b_{c}}\right]+n\left[\delta_{p}(1-\eta) \frac{\partial K}{\partial b_{p}}+\frac{\partial V_{2}^{p}}{\partial b_{p}}\right]
$$

Using (8) to replace $\partial V_{2}^{c} / \partial b_{c},(11)$ to replace $\partial V_{2}^{p} / \partial b_{p}, \partial K / \partial b_{p}=\partial K / \partial b_{c}=0$, and (5) updated one period, we obtain (23).

Proof of Proposition 2. Derivation of (27). Since the cash constraint of active and passive consumers in the goods market is binding, we have $m_{c}+d_{m}=p \hat{q}$ for an active consumer and $m_{c}=p q$ for a passive consumer. Furthermore, because active producers are cash-constrained in the secondary bond market, we have $m_{p}=d_{m}=M$. Using $m_{p}=m_{c}=M$ and rearranging terms, we obtain $(27)$.

Derivation of (28). The marginal value of money of an agent at the beginning of a period 
can be written as

$$
\frac{\partial V_{1}}{\partial m}=(1-n)\left[\delta \eta \frac{\partial K}{\partial m_{c}}+\frac{\partial V_{2}^{c}}{\partial m_{c}}\right]+n\left[\delta_{p}(1-\eta) \frac{\partial K}{\partial m_{p}}+\frac{\partial V_{2}^{p}}{\partial m_{p}}\right]
$$

where we used (17). We can use the envelope condition of a consumer in the goods market, (8), to replace $\partial V_{2}^{c} / \partial m_{c}$ and (11) to replace $\partial V_{2}^{p} / \partial m_{p}$. In a symmetric equilibrium, we have $\partial K / \partial m_{c}=\phi u^{\prime}(\hat{q})-\phi u^{\prime}(q)$ and $\partial K / \partial m_{p}=\phi u^{\prime}(\hat{q})-\phi$, because $\partial \hat{q} / \partial m_{c}=\partial q / \partial m_{c}=1 / p=\phi$, $\partial d_{m} / \partial m_{c}=0$, and $\partial \hat{q} / \partial m_{p}=\phi, \partial q / \partial m_{p}=0$, and $\partial d_{m} / \partial m_{p}=1$ with (15). Using these expressions to replace $\partial K / \partial m_{c}$ and $\partial K / \partial m_{p}$ and (5) updated one period, we obtain (22). ${ }^{30}$

Derivation of (29). The derivation of (29) is equal to the derivation of (23) and is not repeated here.

Proof of Proposition 3. Derivation of (33). Since the bond constraint of active consumers is binding in the secondary bond market, we have $d_{b}=b$. Since the cash constraint of active and passive consumers is binding in the goods market, we have $m_{c}+d_{m}=p \hat{q}$ and $m_{c}=p q$. Using these expressions and the first-order condition of producers in the goods market, $p \phi=1$, in (13), we obtain (33).

Derivation of (34). The marginal value of money of an agent at the beginning of a period can be written as

$$
\frac{\partial V_{1}}{\partial m}=(1-n)\left[\delta \eta \frac{\partial K}{\partial m_{c}}+\frac{\partial V_{2}^{c}}{\partial m_{c}}\right]+n\left[\delta_{p}(1-\eta) \frac{\partial K}{\partial m_{p}}+\frac{\partial V_{2}^{p}}{\partial m_{p}}\right]
$$

where we used (17). We can use the envelope condition of a consumer in the goods market, (8), to replace $\partial V_{2}^{c} / \partial m_{c}$ and (11) to replace $\partial V_{2}^{p} / \partial m_{p}$. Furthermore, we can use $\partial K / \partial m_{p}=0$, because $\partial \hat{q} / \partial m_{p}=\partial q / \partial m_{p}=\partial d_{m} / \partial m_{p}=0$ with (15), ${ }^{31}$ to obtain

$$
\frac{\partial V_{1}}{\partial m}=(1-n)\left[\delta \eta \frac{\partial K}{\partial m_{c}}+\phi u^{\prime}(q)\right]+n \phi .
$$

Next, we need to derive $\partial K / \partial m_{c}$. By (15), we have

$$
\frac{\partial K}{\partial m_{c}}=u^{\prime}(\hat{q}) \frac{\partial \hat{q}}{\partial m_{c}}-u^{\prime}(q) \frac{\partial q}{\partial m_{c}}-\phi \frac{\partial d_{m}}{\partial m_{c}} .
$$

Next, we have to find $\partial \hat{q} / \partial m_{c}$ and $\partial d_{m} / \partial m_{c}$. By (13), we have

$$
(1-\eta)\left[u^{\prime}(\hat{q}) \frac{\partial \hat{q}}{\partial m_{c}}-u^{\prime}(q) \frac{\partial q}{\partial m_{c}}\right]=-\eta \phi \frac{\partial d_{m}}{\partial m_{c}}
$$

\footnotetext{
${ }^{30}$ Since $\hat{q}=\phi\left(m_{c}+d_{m}\right)=\phi\left(m_{c}+m_{p}\right)$ and $q=\phi m_{p}, \partial \hat{q} / \partial m_{c}=\partial \hat{q} / \partial m_{p}=\partial q / \partial m_{c}=\phi$. Since $d_{m}=m_{p}$ is binding, equation (24) holds. Thus, if a buyer has a little more (or less) $m_{c}$, agents will still trade $d_{m}=m_{p}$. Hence $\partial d_{m} / \partial m_{c}=0$. If a producer has a little more (or less) $m_{p}$, then he will still trade the whole $m_{p}$. Hence $\partial d_{m} / \partial m_{p}=1 . \partial q / \partial m_{p}=0$ is obvious.

${ }^{31}$ It is obvious that $\partial \hat{q} / \partial m_{p}=\partial q / \partial m_{p}=\partial d_{m} / \partial m_{p}=0$, since $\hat{q}, q$, and $d_{m}$ do not depend on the producer's money holdings, because the producer's cash constraint is non-binding in the type-III equilibrium.
} 
and since $\hat{q}=\phi\left(m_{c}+d_{m}\right), q=\phi m_{c}$, we also have

$$
\frac{\partial \hat{q}}{\partial m_{c}}=\phi+\phi \frac{\partial d_{m}}{\partial m_{c}} \quad \text { and } \quad \frac{\partial q}{\partial m_{c}}=\phi .
$$

By solving the system of equations (42) and (43), we can derive

$$
\begin{aligned}
\frac{\partial \hat{q}}{\partial m_{c}} & =\phi \frac{\eta+(1-\eta) u^{\prime}(q)}{\eta+(1-\eta) u^{\prime}(\hat{q})} \\
\frac{\partial d_{m}}{\partial m_{c}} & =-\frac{1-\eta}{\phi \eta}\left[u^{\prime}(\hat{q}) \frac{\partial \hat{q}}{\partial m_{c}}-u^{\prime}(q) \frac{\partial q}{\partial m_{c}}\right] .
\end{aligned}
$$

Using (44) and (45) in (41), we have

$$
\begin{aligned}
\frac{\partial K}{\partial m_{c}} & =u^{\prime}(\hat{q}) \frac{\partial \hat{q}}{\partial m_{c}}-u^{\prime}(q) \frac{\partial q}{\partial m_{c}}+\frac{1-\eta}{\eta}\left[u^{\prime}(\hat{q}) \frac{\partial \hat{q}}{\partial m_{c}}-u^{\prime}(q) \frac{\partial q}{\partial m_{c}}\right] \\
& =\frac{1}{\eta}\left[u^{\prime}(\hat{q}) \frac{\partial \hat{q}}{\partial m_{c}}-u^{\prime}(q) \frac{\partial q}{\partial m_{c}}\right]=\frac{1}{\eta}\left[\phi u^{\prime}(\hat{q}) \frac{\eta+(1-\eta) u^{\prime}(q)}{\eta+(1-\eta) u^{\prime}(\hat{q})}-\phi u^{\prime}(q)\right],
\end{aligned}
$$

where we have used $\partial q / \partial m_{c}=\phi$. Using the above equation in (40) and (5) updated one period, we obtain (34).

Derivation of (35). The marginal value of bonds at the beginning of a period can be written as

$$
\frac{\partial V_{1}}{\partial b}=(1-n)\left[\delta \eta \frac{\partial K}{\partial b_{c}}+\frac{\partial V_{2}^{c}}{\partial b_{c}}\right]+n\left[\delta_{p}(1-\eta) \frac{\partial K}{\partial b_{p}}+\frac{\partial V_{2}^{p}}{\partial b_{p}}\right] .
$$

By (33), we can find

$$
\frac{\partial \hat{q}}{\partial b}=\frac{\phi}{(1-\eta) u^{\prime}(\hat{q})+\eta} \quad \text { and } \quad \frac{\partial d_{m}}{\partial b}=\frac{1}{(1-\eta) u^{\prime}(\hat{q})+\eta}
$$

since $\mathcal{B}=\phi b / \phi m=\phi b / q$. Using the above equations to replace $\partial \hat{q} / \partial b_{c}$ and $\partial d_{m} / \partial b_{c}, \partial q / \partial b_{c}=0$, and $\partial \hat{q} / \partial b_{p}=\partial q / \partial b_{p}=\partial d_{m} / \partial b_{p}=\partial q / \partial b_{c}=0$, we obtain

$$
\begin{aligned}
& \frac{\partial K}{\partial b_{c}}=\phi \frac{u^{\prime}(\hat{q})-1}{(1-\eta) u^{\prime}(\hat{q})+\eta}, \\
& \frac{\partial K}{\partial b_{p}}=0 .
\end{aligned}
$$

Using the two above equations in (46), (8) to replace $\partial V_{2}^{c} / \partial b_{c},(11)$ to replace $\partial V_{2}^{p} / \partial b_{p}$, and (5) updated one period, we obtain (35).

Proof of Proposition 4. In the type-I equilibrium, agents must hold enough money and bonds to support the optimal amount of consumption for active consumers, $u^{\prime}(\hat{q})=1$. First, consider 
the following system of equations

$$
\begin{aligned}
u^{\prime}(\hat{q}) & =1, \\
\hat{q} & =2 q, \\
\frac{\gamma}{\beta} & =(1-n) \delta \eta+(1-n)(1-\delta \eta) u^{\prime}(q)+n,
\end{aligned}
$$

with three variables $\hat{q}, q$, and $\gamma$. Let $\hat{q}_{12}, q_{12}$, and $\gamma_{12}$ be the solution of the system of equations. The existence of the solution is immediate. By construction, $\gamma_{12}$ is the threshold of the inflation rate at which the cash constraint of active producers is just binding, $\phi m_{p}=\phi d_{m}$. From the proof of proposition 2, we know that $\hat{q}=2 q$. Hence $\gamma \leq \gamma_{12}$ must hold in the type-I equilibrium.

Furthermore, the bond-to-money ratio must be high enough in order to support $u^{\prime}(\hat{q})=1$. Hence, for a given $\gamma \leq \gamma_{12}$, consider the following system of equations

$$
\begin{aligned}
u^{\prime}(\hat{q}) & =1, \\
\mathcal{B} q & =(1-\eta)[u(\hat{q})-u(q)]+\eta(\hat{q}-q), \\
\frac{\gamma}{\beta} & =(1-n) \delta \eta+(1-n)(1-\delta \eta) u^{\prime}(q)+n,
\end{aligned}
$$

with three variables $\hat{q}, q$, and $\mathcal{B}$. Let $\hat{q}_{13}(\gamma), q_{13}(\gamma)$, and $\mathcal{B}_{13}(\gamma)$ be the solution of the system of equations. The existence of the solution is immediate, since $u^{\prime}$ is continuous and $u^{\prime}(\infty)=0$. By construction, $\mathcal{B}_{13}(\gamma)$ is the threshold of the bond-to-money ratio at which the bond constraint of active consumers is just binding. Hence, $\mathcal{B} \geq \mathcal{B}_{13}(\gamma)$ must hold in the type-I equilibrium.

Proof of Proposition 5. First, consider the following system of equations,

$$
\begin{aligned}
u^{\prime}(\hat{q}) & =1, \\
\hat{q} & =2 q, \\
\frac{\gamma}{\beta} & =(1-n) \delta u^{\prime}(\hat{q})+(1-n)(1-\delta \eta) u^{\prime}(q)-(1-n) \delta(1-\eta)+n,
\end{aligned}
$$

with three variables $\hat{q}, q$, and $\gamma$. Let $\hat{q}_{21}, q_{21}$, and $\gamma_{21}$ be the solution of the system of equations. With $u^{\prime}(\hat{q})=1$, we have

$$
\frac{\gamma}{\beta}=(1-n)(1-\delta \eta) u^{\prime}(q)+(1-n) \delta \eta+n
$$

Hence the solution exists, and $\gamma_{21}=\gamma_{12}$. By the construction of $\gamma_{21}=\gamma_{12}$, it follows that $\gamma_{12}$ is the threshold of the inflation rate at which active consumers can consume the optimal amount of goods, $u^{\prime}(\hat{q})=1$. Hence $\gamma \geq \gamma_{12}$ must hold in the type-II equilibrium.

In the type-II equilibrium, the bond-to-money ratio must be high enough such that the bond constraint is not binding in the secondary bond market. Hence, given that $\gamma \geq \gamma_{12}$, we consider 
the following system of equations

$$
\begin{aligned}
\hat{q} & =2 q, \\
\mathcal{B} q & =(1-\eta)[u(\hat{q})-u(q)]+\eta(\hat{q}-q), \\
\frac{\gamma}{\beta} & =(1-n) \delta u^{\prime}(\hat{q})+(1-n)(1-\delta \eta) u^{\prime}(q)-(1-n) \delta(1-\eta)+n,
\end{aligned}
$$

with three variables $\hat{q}, q$, and $\mathcal{B}$. Let $\hat{q}_{23}(\gamma), q_{23}(\gamma)$, and $\mathcal{B}_{23}(\gamma)$ be the solution of the system of equations. The existence of the solution is immediate, since $u^{\prime}$ is continuous and $u^{\prime}(0)=\infty$. By construction, $\mathcal{B}_{23}(\gamma)$ is the threshold of the bond-to-money ratio at which the bond constraint is just binding conditional on the given inflation rate $\gamma$. Hence $\mathcal{B}>\mathcal{B}_{13}(\gamma)$ must hold in the type-II equilibrium.

Proof of Proposition 6. Case $\gamma \leq \gamma_{12}$ : Given $\gamma \leq \gamma_{12}$, consider the following system of equations,

$$
\begin{aligned}
u^{\prime}(\hat{q}) & =1, \\
\mathcal{B} q & =(1-\eta)[u(\hat{q})-u(q)]+\eta(\hat{q}-q), \\
\frac{\gamma}{\beta} & =(1-n) \delta u^{\prime}(\hat{q}) \frac{\eta+(1-\eta) u^{\prime}(q)}{\eta+(1-\eta) u^{\prime}(\hat{q})}+(1-n)(1-\delta) u^{\prime}(q)+n,
\end{aligned}
$$

with three variables $\hat{q}, q$, and $\mathcal{B}$. Let $\hat{q}_{31}(\gamma), q_{31}(\gamma)$, and $\mathcal{B}_{31}(\gamma)$ be the solution of the system of equations. With $u^{\prime}(\hat{q})=1$, we have

$$
\frac{\gamma}{\beta}=(1-n)(1-\delta \eta) u^{\prime}(q)+(1-n) \delta \eta+n
$$

Hence the solution exists, and $\mathcal{B}_{31}(\gamma)=\mathcal{B}_{13}(\gamma)$. By the construction of $\mathcal{B}_{31}(\gamma)=\mathcal{B}_{13}(\gamma)$, it follows that $\mathcal{B}_{13}(\gamma)$ is the threshold of the bond-to-money ratio at which active consumers can consume the optimal amount of goods $u^{\prime}(\hat{q})=1$. Hence, $\mathcal{B} \leq \mathcal{B}_{13}(\gamma)$ must hold in the type-III equilibrium conditional on $\gamma \leq \gamma_{12}$.

Case $\gamma>\gamma_{12}$ : Given $\gamma>\gamma_{12}$, consider the system of equations,

$$
\begin{aligned}
\hat{q} & =2 q, \\
\mathcal{B} q & =(1-\eta)[u(\hat{q})-u(q)]+\eta(\hat{q}-q), \\
\frac{\gamma}{\beta} & =(1-n) \delta u^{\prime}(\hat{q}) \frac{\eta+(1-\eta) u^{\prime}(q)}{\eta+(1-\eta) u^{\prime}(\hat{q})}+(1-n)(1-\delta) u^{\prime}(q)+n
\end{aligned}
$$

with three variables $\hat{q}, q$, and $\mathcal{B}$. Let $\hat{q}_{32}(\gamma), q_{32}(\gamma)$, and $\mathcal{B}_{32}(\gamma)$ be the solution of the system of equations. The existence of the solution is immediate, since $u^{\prime}$ is continuous, $\lim _{q \rightarrow 0} \frac{(1-n) \delta u^{\prime}(2 q)}{\eta+(1-\eta) u^{\prime}(2 q)}=$ $\frac{(1-n) \delta}{(1-\eta)} \in \mathbb{R}$, and $u^{\prime}(0)=\infty$. By construction, $\mathcal{B}_{32}(\gamma)$ is the threshold of the bond-to-money ratio at which the cash constraint in the secondary bond market is also binding. Hence, $\mathcal{B}<\mathcal{B}_{32}(\gamma)$ must hold in the type-III equilibrium conditional on $\gamma>\gamma_{12}$. 
Proof of Lemma 7. (i): Note that $\hat{q}_{13}(\gamma), q_{13}(\gamma)$, and $\mathcal{B}_{13}(\gamma)$ are the solutions of the system of equations

$$
\begin{aligned}
u^{\prime}(\hat{q}) & =1 \\
\mathcal{B} q & =(1-\eta)[u(\hat{q})-u(q)]+\eta(\hat{q}-q), \\
\frac{\gamma}{\beta} & =(1-n) \delta \eta+(1-n)(1-\delta \eta) u^{\prime}(q)+n
\end{aligned}
$$

From the last equation, it is easy to see that $q_{13}$ is decreasing in $\gamma$. From the first and the second equation, we have

$$
\frac{\partial \mathcal{B}}{\partial q}=\frac{(1-\eta)\left(-u^{\prime}(q)-\eta\right)}{q}-\frac{(1-\eta)[u(\hat{q})-u(q)]+\eta(\hat{q}-q)}{q^{2}}<0 .
$$

Therefore, as $\gamma$ increases, $q_{13}$ decreases and $\mathcal{B}_{13}(\gamma)$ increases.

(ii): Note that $\hat{q}_{23}(\gamma), q_{23}(\gamma)$, and $\mathcal{B}_{23}(\gamma)$ are the solutions of the system of equations

$$
\begin{aligned}
\hat{q} & =2 q, \\
\mathcal{B} q & =(1-\eta)[u(\hat{q})-u(q)]+\eta(\hat{q}-q), \\
\frac{\gamma}{\beta} & =(1-n) \delta u^{\prime}(\hat{q})+(1-n)(1-\delta \eta) u^{\prime}(q)-(1-n) \delta(1-\eta)+n .
\end{aligned}
$$

From the first and the last equation, it is easy to see that $q_{23}$ is decreasing in $\gamma$. From the first and the second equation, we have

$$
\frac{q^{2}}{1-\eta} \frac{\partial \mathcal{B}}{\partial q}=\left[2 q u^{\prime}(2 q)-q u^{\prime}(q)\right]-[u(2 q)-u(q)]=\left[2 q u^{\prime}(2 q)-u(2 q)\right]-\left[q u^{\prime}(q)-u(q)\right] .
$$

Note that $x u^{\prime}(x)-u(x)$ is decreasing in $x$, since its derivative is

$$
u^{\prime}(x)+u^{\prime \prime}(x)-u^{\prime}(x)<0 .
$$

Hence $\partial \mathcal{B} / \partial q<0$. Therefore, as $\gamma$ increases, $q_{23}$ decreases and $\mathcal{B}_{23}(\gamma)$ increases.

(iii): Note that $\hat{q}_{32}(\gamma), q_{32}(\gamma)$, and $\mathcal{B}_{32}(\gamma)$ are the solution of the system of equations

$$
\begin{aligned}
\hat{q} & =2 q, \\
\mathcal{B} q & =(1-\eta)[u(\hat{q})-u(q)]+\eta(\hat{q}-q), \\
\frac{\gamma}{\beta} & =(1-n) \delta u^{\prime}(\hat{q}) \frac{\eta+(1-\eta) u^{\prime}(q)}{\eta+(1-\eta) u^{\prime}(\hat{q})}+(1-n)(1-\delta) u^{\prime}(q)+n .
\end{aligned}
$$

From the first and the last equation, we have

$$
\frac{\gamma}{\beta}=(1-n) \delta u^{\prime}(2 q) \frac{\eta+(1-\eta) u^{\prime}(q)}{\eta+(1-\eta) u^{\prime}(2 q)}+(1-n)(1-\delta) u^{\prime}(q)+n
$$


If we differentiate the right-hand side of the above equation, then we obtain

$$
\begin{aligned}
& 2(1-n) \delta u^{\prime \prime}(2 q) \frac{\eta+(1-\eta) u^{\prime}(q)}{\eta+(1-\eta) u^{\prime}(2 q)}+(1-n)(1-\delta) u^{\prime \prime}(q) \\
& +(1-n) \delta u^{\prime}(2 q) \frac{(1-\eta) u^{\prime \prime}(q)}{\eta+(1-\eta) u^{\prime}(2 q)}-(1-n) \delta u^{\prime}(2 q) \frac{2\left[\eta+(1-\eta) u^{\prime}(q)\right](1-\eta) u^{\prime \prime}(2 q)}{\left[\eta+(1-\eta) u^{\prime}(2 q)\right]^{2}}
\end{aligned}
$$

Multiplying the above formula by $\left[\eta+(1-\eta) u^{\prime}(2 q)\right]^{2}$, we obtain

$$
\begin{aligned}
& \left.2(1-n) \delta u^{\prime \prime \prime}(q)\right]\left[\eta+(1-\eta) u^{\prime}(2 q)\right] \\
& \left.+(1-n)(1-\delta) u^{\prime \prime \prime}(2 q)\right]^{2} \\
& \left.+(1-n) \delta u^{\prime}(2 q)(1-\eta) u^{\prime \prime \prime}(2 q)\right] \\
& -2(1-n) \delta u^{\prime}(2 q)\left[\eta+(1-\eta) u^{\prime}(q)\right](1-\eta) u^{\prime \prime}(2 q) \\
& \left.=2(1-n) \delta u^{\prime \prime \prime}(q)\right] \eta \\
& \left.+(1-n)(1-\delta) u^{\prime \prime \prime}(2 q)\right]^{2} \\
& \left.+(1-n) \delta u^{\prime}(2 q)(1-\eta) u^{\prime \prime \prime}(2 q)\right]<0 .
\end{aligned}
$$

The last inequality follows immediately from $u^{\prime \prime}<0$. Hence, $q_{32}$ is decreasing in $\gamma$. From the first and the second equation, we have

$$
\frac{\partial \mathcal{B}}{\partial q}<0
$$

as is shown in (ii). Therefore, as $\gamma$ increases, $q_{32}$ decreases and $\mathcal{B}_{32}(\gamma)$ increases.

(iv): The proof is immediate by noticing that the following three formulas are identical when $u^{\prime}(\hat{q})=1$ :

$$
\begin{aligned}
& (1-n) \delta \eta+(1-n)(1-\delta \eta) u^{\prime}(q)+n \\
& =(1-n) \delta u^{\prime}(\hat{q})+(1-n)(1-\delta \eta) u^{\prime}(q)-(1-n) \delta(1-\eta)+n \\
& =(1-n) \delta u^{\prime}(\hat{q}) \frac{\eta+(1-\eta) u^{\prime}(q)}{\eta+(1-\eta) u^{\prime}(\hat{q})}+(1-n)(1-\delta) u^{\prime}(q)+n
\end{aligned}
$$

Proof of Lemma 8. Given $\gamma>\gamma_{12}$, we have

$$
\begin{aligned}
\frac{\gamma}{\beta} & =(1-n) \delta u^{\prime}\left(2 q_{23}(\gamma)\right)+(1-n)(1-\delta \eta) u^{\prime}\left(q_{23}(\gamma)\right)-(1-n) \delta(1-\eta)+n \\
& =(1-n) \delta u^{\prime}\left(2 q_{32}(\gamma)\right) \frac{\eta+(1-\eta) u^{\prime}\left(q_{32}(\gamma)\right)}{\eta+(1-\eta) u^{\prime}\left(2 q_{32}(\gamma)\right)}+(1-n)(1-\delta) u^{\prime}\left(q_{32}(\gamma)\right)+n
\end{aligned}
$$


Let

$$
\begin{aligned}
f_{2}(q) & \equiv(1-n) \delta u^{\prime}(2 q)+(1-n)(1-\delta \eta) u^{\prime}(q)-(1-n) \delta(1-\eta)+n \\
f_{3}(q) & \equiv(1-n) \delta u^{\prime}(2 q) \frac{\eta+(1-\eta) u^{\prime}(q)}{\eta+(1-\eta) u^{\prime}(2 q)}+(1-n)(1-\delta) u^{\prime}(q)+n
\end{aligned}
$$

then

$$
f_{2}\left(q_{23}(\gamma)\right)=f_{3}\left(q_{32}(\gamma)\right)=\frac{\gamma}{\beta}
$$

Note that

$$
f_{2}(q)-f_{3}(q)=\frac{(1-n) \delta(1-\eta)}{\eta+(1-\eta) u^{\prime}(2 q)}\left[u^{\prime}(2 q)-1\right]\left[u^{\prime}(2 q)-\eta u^{\prime}(q)+\eta\right] .
$$

Since by assumption $u^{\prime}(2 q)-\eta u^{\prime}(q)+\eta>0$, we have $f_{2}(q)>f_{3}(q)$. Moreover, because

$$
\frac{\partial f_{2}}{\partial q}=2(1-n) \delta u^{\prime \prime}(2 q)+(1-n)(1-\delta \eta) u^{\prime \prime}(q)<0,
$$

we must have

$$
q_{23}(\gamma)>q_{32}(\gamma)
$$

Otherwise, we would have a contradiction, $\gamma / \beta=f_{2}\left(q_{23}(\gamma)\right)>f_{3}\left(q_{32}(\gamma)\right)=\gamma / \beta$.

By (13), the bond-to-money ratio is

$$
\mathcal{B}=(1-\eta) \frac{u(2 q)-u(q)}{q}+\eta
$$

Hence, if we differentiate $\mathcal{B}$ with respect to $q$, we obtain

$$
\frac{\partial \mathcal{B}}{\partial q}=\frac{2 u^{\prime}(2 q)-u^{\prime}(q)}{q}-\frac{u(2 q)-u(q)}{q^{2}}<0,
$$

as we showed in the proof of Lemma 7 . Therefore $\mathcal{B}_{32}(\gamma)>\mathcal{B}_{23}(\gamma)$, because $q_{23}(\gamma)>q_{32}(\gamma)$.

Proof of Lemma 9. As $\gamma>\gamma_{12}$ approaches $\gamma_{12}, q_{32}(\gamma)<q^{*} / 2$ approaches $q^{*} / 2$. Hence, for a given $a$, we have $q_{32}(\gamma)>a$ for a sufficiently small $\gamma$. From the previous proof, we have

$$
f_{2}(q)-f_{3}(q)=\frac{(1-n) \delta(1-\eta)}{\eta+(1-\eta) u^{\prime}(2 q)}\left[u^{\prime}(2 q)-1\right]\left[u^{\prime}(2 q)-\eta u^{\prime}(q)+\eta\right] .
$$

For a small $\gamma$, so for $q_{32}(\gamma)>a$, we have $u^{\prime}\left(2 q_{32}(\gamma)\right)-\eta u^{\prime}\left(q_{32}(\gamma)\right)+\eta>0$. Therefore, it holds that $f_{2}\left(q_{32}(\gamma)\right)>f_{3}\left(q_{32}(\gamma)\right)$. Because also $\partial f_{2} / \partial q<0$, and

$$
\frac{\gamma}{\beta}=f_{2}\left(q_{23}(\gamma)\right)=f_{3}\left(q_{32}(\gamma)\right)=\frac{\gamma}{\beta},
$$

we must have

$$
q_{23}(\gamma)>q_{32}(\gamma)
$$


Otherwise, we would have a contradiction, $\gamma / \beta=f_{2}\left(q_{23}(\gamma)\right)>f_{3}\left(q_{32}(\gamma)\right)=\gamma / \beta$. At last, we have

$$
\frac{\partial \mathcal{B}}{\partial q}=\frac{2 u^{\prime}(2 q)-u^{\prime}(q)}{q}-\frac{u(2 q)-u(q)}{q^{2}}<0 .
$$

Since $q_{23}(\gamma)>q_{32}(\gamma)>a$, we must have $\mathcal{B}_{32}(\gamma)>\mathcal{B}_{23}(\gamma)$.

Proof of Lemma 10. As $\gamma$ approaches $\infty, q_{32}(\gamma)$ approaches zero. Hence for a given $a$, $q_{32}(\gamma)<a$ for a sufficiently large $\gamma$. From the previous proof, we have

$$
f_{2}(q)-f_{3}(q)=\frac{(1-n) \delta(1-\eta)}{\eta+(1-\eta) u^{\prime}(2 q)}\left[u^{\prime}(2 q)-1\right]\left[u^{\prime}(2 q)-\eta u^{\prime}(q)+\eta\right] .
$$

For large $\gamma$, i.e., for $q_{32}(\gamma)<a$, we have $u^{\prime}\left(2 q_{32}(\gamma)\right)-\eta u^{\prime}\left(q_{32}(\gamma)\right)+\eta<0$. Therefore, it holds that $f_{2}\left(q_{32}(\gamma)\right)<f_{3}\left(q_{32}(\gamma)\right)$. Because also $\partial f_{2} / \partial q<0$, and

$$
\frac{\gamma}{\beta}=f_{2}\left(q_{23}(\gamma)\right)=f_{3}\left(q_{32}(\gamma)\right)=\frac{\gamma}{\beta}
$$

we must have

$$
q_{23}(\gamma)<q_{32}(\gamma)
$$

Otherwise, we would have a contradiction, $\gamma / \beta=f_{2}\left(q_{23}(\gamma)\right)<f_{3}\left(q_{32}(\gamma)\right)=\gamma / \beta$. At last, we have

$$
\frac{\partial \mathcal{B}}{\partial q}=\frac{2 u^{\prime}(2 q)-u^{\prime}(q)}{q}-\frac{u(2 q)-u(q)}{q^{2}}<0 .
$$

Since $q_{23}(\gamma)<q_{32}(\gamma)<a$, we must have $\mathcal{B}_{32}(\gamma)<\mathcal{B}_{23}(\gamma)$.

Proof of Proposition 11. Consider any $(\gamma, \mathcal{B})$ that supports the type-III equilibrium. Note that $\hat{q}=\hat{q}(\gamma, \mathcal{B}), q=q(\gamma, \mathcal{B})$, and $q_{p}=q_{p}(\gamma, \mathcal{B})$ are completely determined by $(\gamma, \mathcal{B})$ in the type-III equilibrium, because $\hat{q}, q$ and $q_{p}$ are solutions of the system of equations,

$$
\begin{aligned}
n q_{p} & =(1-n)[\delta \hat{q}+(1-\delta) q] \\
\mathcal{B} q & =(1-\eta)[u(\hat{q})-u(q)]+\eta(\hat{q}-q), \\
\frac{\gamma}{\beta} & =(1-n) \delta u^{\prime}(\hat{q}) \frac{\eta+(1-\eta) u^{\prime}(q)}{\eta+(1-\eta) u^{\prime}(\hat{q})}+(1-n)(1-\delta) u^{\prime}(q)+n
\end{aligned}
$$

By differentiating equation (47) with respect to $\mathcal{B}$, we obtain

$$
n \frac{\partial q_{p}}{\partial \mathcal{B}}=(1-n)\left[\delta \frac{\partial \hat{q}}{\partial \mathcal{B}}+(1-\delta) \frac{\partial q}{\partial \mathcal{B}}\right]
$$

Hence, we can simplify (37) as

$$
(1-\beta) \frac{\partial \mathcal{W}}{\partial \mathcal{B}}=(1-n)\left\{\delta\left[u^{\prime}(\hat{q})-1\right] \frac{\partial \hat{q}}{\partial \mathcal{B}}+(1-\delta)\left[u^{\prime}(q)-1\right] \frac{\partial q}{\partial \mathcal{B}}\right\}
$$


By differentiating equation (48) with respect to $\mathcal{B}$, we have

$$
q+\mathcal{B} \frac{\partial q}{\partial \mathcal{B}}=(1-\eta)\left[u^{\prime}(\hat{q}) \frac{\partial \hat{q}}{\partial \mathcal{B}}-u^{\prime}(q) \frac{\partial q}{\partial \mathcal{B}}\right]+\eta\left(\frac{\partial \hat{q}}{\partial \mathcal{B}}-\frac{\partial q}{\partial \mathcal{B}}\right)
$$

i.e., by rearranging the above equation we obtain

$$
\frac{\partial \hat{q}}{\partial \mathcal{B}}=\frac{q}{\eta+(1-\eta) u^{\prime}(\hat{q})}+\frac{\mathcal{B}+\eta+(1-\eta) u^{\prime}(q)}{\eta+(1-\eta) u^{\prime}(\hat{q})} \frac{\partial q}{\partial \mathcal{B}} .
$$

By differentiating equation (49) with respect to $\mathcal{B}$, we have

$$
\begin{aligned}
0 & =(1-n) \delta u^{\prime \prime}(\hat{q}) \frac{\partial \hat{q}}{\partial \mathcal{B}} \frac{\eta+(1-\eta) u^{\prime}(q)}{\eta+(1-\eta) u^{\prime}(\hat{q})}+(1-n) \delta u^{\prime}(\hat{q}) \frac{(1-\eta) u^{\prime \prime}(q)}{\eta+(1-\eta) u^{\prime}(\hat{q})} \frac{\partial q}{\partial \mathcal{B}} \\
& -(1-n) \delta u^{\prime}(\hat{q}) \frac{\eta+(1-\eta) u^{\prime}(q)}{\left[\eta+(1-\eta) u^{\prime}(\hat{q})\right]^{2}}(1-\eta) u^{\prime \prime}(\hat{q}) \frac{\partial \hat{q}}{\partial \mathcal{B}}+(1-n)(1-\delta) u^{\prime \prime}(q) \frac{\partial q}{\partial \mathcal{B}} .
\end{aligned}
$$

With the equations (50) and (51), we have

$$
\begin{aligned}
0 & =(1-n) \delta u^{\prime \prime}(\hat{q})\left[\frac{q}{\eta+(1-\eta) u^{\prime}(\hat{q})}+\frac{\mathcal{B}+\eta+(1-\eta) u^{\prime}(q)}{\eta+(1-\eta) u^{\prime}(\hat{q})} \frac{\partial q}{\partial \mathcal{B}}\right] \frac{\eta+(1-\eta) u^{\prime}(q)}{\eta+(1-\eta) u^{\prime}(\hat{q})} \\
& +(1-n) \delta u^{\prime}(\hat{q}) \frac{(1-\eta) u^{\prime \prime}(q)}{\eta+(1-\eta) u^{\prime}(\hat{q})} \frac{\partial q}{\partial \mathcal{B}} \\
& -(1-n) \delta u^{\prime}(\hat{q}) \frac{\eta+(1-\eta) u^{\prime}(q)}{\left[\eta+(1-\eta) u^{\prime}(\hat{q})\right]^{2}}(1-\eta) u^{\prime \prime}(\hat{q})\left[\frac{q}{\eta+(1-\eta) u^{\prime}(\hat{q})}+\frac{\mathcal{B}+\eta+(1-\eta) u^{\prime}(q)}{\eta+(1-\eta) u^{\prime}(\hat{q})} \frac{\partial q}{\partial \mathcal{B}}\right] \\
& +(1-n)(1-\delta) u^{\prime \prime}(q) \frac{\partial q}{\partial \mathcal{B}} .
\end{aligned}
$$

By factoring $\partial q / \partial \mathcal{B}$, we have

$$
\left.\begin{array}{l}
-(1-n) \delta u^{\prime \prime}(\hat{q}) \frac{q}{\eta+(1-\eta) u^{\prime}(\hat{q})} \frac{\eta+(1-\eta) u^{\prime}(q)}{\eta+(1-\eta) u^{\prime}(\hat{q})} \\
+(1-n) \delta u^{\prime}(\hat{q}) \frac{\eta+(1-\eta) u^{\prime}(q)}{\left[\eta+(1-\eta) u^{\prime}(\hat{q})\right]^{2}}(1-\eta) u^{\prime \prime}(\hat{q}) \frac{q}{\eta+(1-\eta) u^{\prime}(\hat{q})} \\
=\left\{\begin{array}{c}
(1-n) \delta u^{\prime \prime}(\hat{q}) \frac{\mathcal{B}+\eta+(1-\eta) u^{\prime}(q)}{\eta+(1-\eta) u^{\prime}(\hat{q})} \frac{\eta+(1-\eta) u^{\prime}(q)}{\eta+(1-\eta) u^{\prime}(\hat{q})} \\
+(1-n) \delta u^{\prime}(\hat{q}) \frac{(1-\eta) u^{\prime \prime}(q)}{\eta+(1-\eta) u^{\prime}(\hat{q})} \\
-(1-n) \delta u^{\prime}(\hat{q}) \frac{\eta+(1-\eta) u^{\prime}(q)}{\left[\eta+(1-\eta) u^{\prime}(\hat{q})\right]^{2}}(1-\eta) u^{\prime \prime}(\hat{q}) \frac{\hat{\mathcal{B}}+\eta+(1-\eta) u^{\prime}(q)}{\eta+(1-\eta) u^{\prime}(\hat{q})} \\
+(1-n)(1-\delta) u^{\prime \prime}(q)
\end{array}\right.
\end{array}\right\} \frac{\partial q}{\partial \mathcal{B}} .
$$


If we multiply the above equation by $\left[\eta+(1-\eta) u^{\prime}(\hat{q})\right]^{3}$, we obtain

$$
\begin{aligned}
& -(1-n) \delta u^{\prime \prime}(\hat{q}) q\left[\eta+(1-\eta) u^{\prime}(q)\right]\left[\eta+(1-\eta) u^{\prime}(\hat{q})\right] \\
& +(1-n) \delta u^{\prime}(\hat{q})\left[\eta+(1-\eta) u^{\prime}(q)\right](1-\eta) u^{\prime \prime}(\hat{q}) q \\
& =\left\{\begin{array}{c}
(1-n) \delta u^{\prime \prime}(\hat{q})\left[\mathcal{B}+\eta+(1-\eta) u^{\prime}(q)\right]\left[\eta+(1-\eta) u^{\prime}(q)\right]\left[\eta+(1-\eta) u^{\prime}(\hat{q})\right] \\
+(1-n) \delta u^{\prime}(\hat{q})(1-\eta) u^{\prime \prime}(q)\left[\eta+(1-\eta) u^{\prime}(\hat{q})\right]^{2} \\
-(1-n) \delta u^{\prime}(\hat{q})\left[\eta+(1-\eta) u^{\prime}(q)\right](1-\eta) u^{\prime \prime}(\hat{q})\left[\mathcal{B}+\eta+(1-\eta) u^{\prime}(q)\right] \\
+(1-n)(1-\delta) u^{\prime \prime}(q)\left[\eta+(1-\eta) u^{\prime}(\hat{q})\right]^{3}
\end{array}\right\} \frac{\partial q}{\partial \mathcal{B}} .
\end{aligned}
$$

After simplifying the above equation, we have

$$
\begin{aligned}
& -(1-n) \delta u^{\prime \prime}(\hat{q}) q\left[\eta+(1-\eta) u^{\prime}(q)\right] \eta \\
& =\left\{\begin{array}{c}
(1-n) \delta u^{\prime \prime}(\hat{q})\left[\mathcal{B}+\eta+(1-\eta) u^{\prime}(q)\right]\left[\eta+(1-\eta) u^{\prime}(q)\right] \eta \\
+(1-n) u^{\prime \prime}(q)\left[\eta+(1-\eta) u^{\prime}(\hat{q})\right]^{2}\left[\eta(1-\delta)+(1-\eta) u^{\prime}(\hat{q})\right]
\end{array}\right\} \frac{\partial q}{\partial \mathcal{B}} .
\end{aligned}
$$

Hence, we can denote

$$
\frac{\partial q}{\partial \mathcal{B}}=\frac{A(\hat{q}, q)}{B(\hat{q}, q, \mathcal{B})}
$$

where

$$
\begin{aligned}
A(\hat{q}, q) & \equiv-(1-n) \delta u^{\prime \prime}(\hat{q}) q\left[\eta+(1-\eta) u^{\prime}(q)\right] \eta \\
B(\hat{q}, q, \mathcal{B}) & \equiv(1-n) \delta u^{\prime \prime}(\hat{q})\left[\mathcal{B}+\eta+(1-\eta) u^{\prime}(q)\right]\left[\eta+(1-\eta) u^{\prime}(q)\right] \eta \\
& +(1-n) u^{\prime \prime}(q)\left[\eta+(1-\eta) u^{\prime}(\hat{q})\right]^{2}\left[\eta(1-\delta)+(1-\eta) u^{\prime}(\hat{q})\right] .
\end{aligned}
$$

Note that since $u^{\prime \prime}<0$, we have

$$
A(\hat{q}, q)>0, \quad \text { and } \quad B(\hat{q}, q, \mathcal{B})<0 .
$$

Hence, $\partial q / \partial \mathcal{B}<0$. Moreover,

$$
\begin{aligned}
{\left[\eta+(1-\eta) u^{\prime}(\hat{q})\right] B(\hat{q}, q, \mathcal{B}) \frac{\partial \hat{q}}{\partial \mathcal{B}} } & =q B(\hat{q}, q, \mathcal{B})+\left[\mathcal{B}+\eta+(1-\eta) u^{\prime}(q)\right] A(\hat{q}, q) \\
& =q(1-n) u^{\prime \prime}(q)\left[\eta+(1-\eta) u^{\prime}(\hat{q})\right]^{2}\left[\eta(1-\delta)+(1-\eta) u^{\prime}(\hat{q})\right]<0
\end{aligned}
$$


Therefore, $\partial \hat{q} / \partial \mathcal{B}>0$. At last, given that

$$
\begin{aligned}
\Theta(\gamma, \mathcal{B}) & \equiv \delta\left[u^{\prime}(\hat{q}(\gamma, \mathcal{B}))-1\right] \frac{\partial \hat{q}}{\partial \mathcal{B}}+(1-\delta)\left[u^{\prime}(q(\gamma, \mathcal{B}))-1\right] \frac{\partial q}{\partial \mathcal{B}} \\
& =\delta\left[u^{\prime}(\hat{q}(\gamma, \mathcal{B}))-1\right] \frac{q(\gamma, \mathcal{B})}{\eta+(1-\eta) u^{\prime}(\hat{q}(\gamma, \mathcal{B}))} \\
& +\left\{\delta\left[u^{\prime}(\hat{q}(\gamma, \mathcal{B}))-1\right] \frac{\mathcal{B}+\eta+(1-\eta) u^{\prime}(q(\gamma, \mathcal{B}))}{\eta+(1-\eta) u^{\prime}(\hat{q}(\gamma, \mathcal{B}))}+(1-\delta)\left[u^{\prime}(q(\gamma, \mathcal{B}))-1\right]\right\} \\
& \times \frac{A(\hat{q}(\gamma, \mathcal{B}), q(\gamma, \mathcal{B}))}{B(\hat{q}(\gamma, \mathcal{B}), q(\gamma, \mathcal{B}), \mathcal{B})},
\end{aligned}
$$

then $(1-\beta) \partial \mathcal{W} / \partial \mathcal{B}=(1-n) \Theta(\gamma, \mathcal{B})$. Thus, if $\Theta(\gamma, \mathcal{B})>0$, then welfare will be improved by increasing $\mathcal{B}$, and if $\Theta(\gamma, \mathcal{B})<0$, then welfare will be improved by decreasing $\mathcal{B}$.

Proof of Theorem 12. By Proposition $4, \gamma<\gamma_{12}$, and $\mathcal{B}>\mathcal{B}_{13}(\gamma)$. Decreasing the bondto-money ratio $\mathcal{B}$ up to $\mathcal{B}_{13}(\gamma)$ will not change welfare, since it supports the type-I equilibrium. But at $\mathcal{B}=\mathcal{B}_{13}(\gamma)$

$$
\Theta\left(\gamma, \mathcal{B}_{13}(\gamma)\right)=(1-\delta)\left[u^{\prime}\left(q\left(\gamma, \mathcal{B}_{13}(\gamma)\right)\right)-1\right] \frac{A\left(\hat{q}\left(\gamma, \mathcal{B}_{13}(\gamma)\right), q\left(\gamma, \mathcal{B}_{13}(\gamma)\right)\right)}{B\left(\hat{q}\left(\gamma, \mathcal{B}_{13}(\gamma)\right), q\left(\gamma, \mathcal{B}_{13}(\gamma)\right), \mathcal{B}_{13}(\gamma)\right)}<0
$$

The inequality comes from $A(\hat{q}, q)>0, B(\hat{q}, q, \mathcal{B})<0$, and $u^{\prime}(q)-1>0$. Welfare will be improved by decreasing $\mathcal{B}$ further at $\mathcal{B}_{13}(\gamma)$ by Proposition 11.

Proof of Proposition 13. Let $u^{\prime}(2 q)-\eta u^{\prime}(q)+\eta>0$ for $q<q^{*} / 2$, and $\gamma>\gamma_{12}$.

First, we show that

$$
\bar{q}_{2}>\bar{q}_{3}, \quad \text { and } \quad \hat{\bar{q}}_{2}>\hat{\bar{q}}_{3},
$$

where $\bar{q}_{2}$ and $\hat{\bar{q}}_{2}$ are the equilibrium outputs at $\mathcal{B}=\mathcal{B}_{32}(\gamma)$ under the type-II equilibrium, and $\bar{q}_{3}$, $\hat{\bar{q}}_{3}$ are the equilibrium outputs at $\mathcal{B}=\mathcal{B}_{32}(\gamma)$ under the type-III equilibrium. By (28) and (34),

$$
\begin{aligned}
& \delta u^{\prime}\left(\hat{\bar{q}}_{2}\right)+(1-\delta \eta) u^{\prime}\left(\bar{q}_{2}\right)-\delta(1-\eta) \\
& =\delta u^{\prime}\left(\hat{\bar{q}}_{3}\right) \frac{\eta+(1-\eta) u^{\prime}\left(\bar{q}_{3}\right)}{\eta+(1-\eta) u^{\prime}\left(\hat{\bar{q}}_{3}\right)}+(1-\delta) u^{\prime}\left(\bar{q}_{3}\right) .
\end{aligned}
$$

By definition, $\hat{\bar{q}}_{2}=2 \bar{q}_{2}$. At $\mathcal{B}=\mathcal{B}_{32}(\gamma)$, we also have $\hat{\bar{q}}_{3}=2 \bar{q}_{3}$. Hence, it is enough to show that if $q_{2}=q_{3}\left(\right.$ and $\left.\hat{q}_{2}=\hat{q}_{3}<q^{*}\right)$, then we have

$$
\begin{aligned}
& \delta u^{\prime}\left(\hat{q}_{2}\right)+(1-\delta \eta) u^{\prime}\left(q_{2}\right)-\delta(1-\eta) \\
& >\delta u^{\prime}\left(\hat{q}_{3}\right) \frac{\eta+(1-\eta) u^{\prime}\left(q_{3}\right)}{\eta+(1-\eta) u^{\prime}\left(\hat{q}_{3}\right)}+(1-\delta) u^{\prime}\left(q_{3}\right) .
\end{aligned}
$$

If so, we must have $\bar{q}_{2}>\bar{q}_{3}$ to satisfy (52), because $u^{\prime \prime}<0$. If $q_{2}=q_{3}$, then equation (52) can be 
simplified (under inequality preserving operations) as

$$
\left[\eta+(1-\eta) u^{\prime}\left(\hat{q}_{2}\right)\right]\left[u^{\prime}\left(q_{2}\right)-1\right]=u^{\prime}\left(\hat{q}_{2}\right)\left[u^{\prime}\left(\bar{q}_{2}\right)-u^{\prime}\left(\hat{q}_{2}\right)\right] .
$$

Note that if $u\left(\hat{q}_{2}\right)=1$, then both terms are equal. If we differentiate both terms with respect to $u\left(\hat{q}_{2}\right)$, then we have

$$
(1-\eta)\left[u^{\prime}\left(q_{2}\right)-1\right], \quad \text { and } \quad u^{\prime}\left(q_{2}\right)-2 u^{\prime}\left(\hat{q}_{2}\right),
$$

in the left-hand side and in the right-hand side, respectively. Since $u^{\prime}\left(\hat{q}_{2}\right)-\eta u^{\prime}(q)+\eta>0$ and $u^{\prime}\left(\hat{q}_{2}\right)>1$, we have

$$
(1-\eta)\left[u^{\prime}\left(q_{2}\right)-1\right]>u^{\prime}\left(q_{2}\right)-2 u^{\prime}\left(\hat{q}_{2}\right)
$$

which implies that

$$
\left[\eta+(1-\eta) u^{\prime}\left(\hat{q}_{2}\right)\right]\left[u^{\prime}\left(q_{2}\right)-1\right]>u^{\prime}\left(\hat{q}_{2}\right)\left[u^{\prime}\left(q_{2}\right)-u^{\prime}\left(\hat{q}_{2}\right)\right] .
$$

Therefore, we find that

$$
\begin{aligned}
& \delta u^{\prime}\left(\hat{q}_{2}\right)+(1-\delta \eta) u^{\prime}\left(q_{2}\right)-\delta(1-\eta) \\
& >\delta u^{\prime}\left(\hat{q}_{3}\right) \frac{\eta+(1-\eta) u^{\prime}\left(q_{3}\right)}{\eta+(1-\eta) u^{\prime}\left(\hat{q}_{3}\right)}+(1-\delta) u^{\prime}\left(q_{3}\right),
\end{aligned}
$$

which consequently implies that

$$
\bar{q}_{2}>\bar{q}_{3}, \text { and } \hat{\bar{q}}_{2}=2 \bar{q}_{2}>2 \bar{q}_{3}=\hat{\bar{q}}_{3} .
$$

Now, we show that

$$
\tilde{q}_{2}>\tilde{q}_{3}, \quad \text { and } \quad \hat{\tilde{q}}_{2}>\hat{\tilde{q}}_{3},
$$

where $\tilde{q}_{2}, \hat{\tilde{q}}_{2}$ are the equilibrium outputs at $\mathcal{B}=\mathcal{B}_{23}(\gamma)$ under the type-II equilibrium, and $\tilde{q}_{3}, \hat{\tilde{q}}_{3}$ are the equilibrium outputs at $\mathcal{B}=\mathcal{B}_{23}(\gamma)$ under the type-III equilibrium. By (23), we have

$$
\mathcal{B}=(1-\eta) \frac{u\left(\hat{\tilde{q}}_{3}\right)-u\left(\tilde{q}_{3}\right)}{\tilde{q}_{3}}+\eta \frac{\hat{\tilde{q}}_{3}-\tilde{q}_{3}}{\tilde{q}_{3}} .
$$

At $\mathcal{B}=\mathcal{B}_{23}(\gamma)$, we also have

$$
\mathcal{B}=(1-\eta) \frac{u\left(\hat{\tilde{q}}_{2}\right)-u\left(\tilde{q}_{2}\right)}{\tilde{q}_{2}}+\eta \frac{\hat{\tilde{q}}_{2}-\tilde{q}_{2}}{\tilde{q}_{2}}
$$

Since

$$
1=\frac{\hat{\tilde{q}}_{2}-\tilde{q}_{2}}{\tilde{q}_{2}}>\frac{\hat{\tilde{q}}_{3}-\tilde{q}_{3}}{\tilde{q}_{3}},
$$

we must have

$$
\frac{u\left(\hat{\tilde{q}}_{3}\right)-u\left(\tilde{q}_{3}\right)}{\tilde{q}_{3}}>\frac{u\left(\hat{\tilde{q}}_{2}\right)-u\left(\tilde{q}_{2}\right)}{\tilde{q}_{2}} .
$$


By the previous result at $\mathcal{B}=\mathcal{B}_{32}(\gamma)$, and $\partial \hat{q} / \partial \mathcal{B}>0$ under the type-III equilibrium from Proposition 11, we have

$$
\hat{\tilde{q}}_{2}>\hat{\tilde{q}}_{3}
$$

Together with the inequality (53), we must have

$$
\tilde{q}_{2}>\tilde{q}_{3}
$$

Lastly, we show that for any $\mathcal{B} \in\left(\mathcal{B}_{23}(\gamma), \mathcal{B}_{32}(\gamma)\right)$,

$$
q_{2}>q_{3}, \quad \text { and } \quad \hat{q}_{2}>\hat{q}_{3},
$$

where $q_{2}, \hat{q}_{2}$ are the equilibrium outputs under the type-II equilibrium, and $q_{3}, \hat{q}_{3}$ are the equilibrium outputs under the type-III equilibrium. By Proposition 11 , we have $\partial q / \partial \mathcal{B}<0$ under the type-III equilibrium. Together with the results at $\mathcal{B}=\mathcal{B}_{23}(\gamma)$, we have

$$
q_{2}>q_{3}, \text { for any } \mathcal{B} \in\left(\mathcal{B}_{23}(\gamma), \mathcal{B}_{32}(\gamma)\right) \text {. }
$$

Since $\hat{q}_{2}=2 q_{2}$ and $\hat{q}_{3}<2 q_{3}$, we also have

$$
\hat{q}_{2}>\hat{q}_{3}
$$

which completes the proof.

Proof of Proposition 14. In the type-III equilibrium, equation (35) holds. Thus, we always have

$$
\frac{\partial \rho}{\partial \mathcal{B}}=\frac{\beta(1-n) \delta \eta}{\gamma} \frac{u^{\prime \prime}(\hat{q}) \frac{\partial \hat{q}}{\partial \mathcal{B}}}{\left[\eta+(1-\eta) u^{\prime}(\hat{q})\right]^{2}}<0
$$

since $\partial \hat{q} / \partial \mathcal{B}>0$.

In the type-III equilibrium, the secondary bond market price satisfies

$$
\varphi^{-1}=(1-\eta) \frac{u(\hat{q})-u(q)}{\hat{q}-q}+\eta,
$$

since $\mathcal{B} q=(1-\eta)[u(\hat{q})-u(q)]+\eta(\hat{q}-q)$ by equation (33). If we differentiate $[u(\hat{q})-u(q)] /(\hat{q}-q)$ with respect to $\mathcal{B}$, then we have

$$
\frac{1}{(\hat{q}-q)}\left\{\left[u^{\prime}(\hat{q}) \frac{\partial \hat{q}}{\partial \mathcal{B}}-u^{\prime}(q) \frac{\partial q}{\partial \mathcal{B}}\right]-\frac{u(\hat{q})-u(q)}{\hat{q}-q}\left(\frac{\partial \hat{q}}{\partial \mathcal{B}}-\frac{\partial q}{\partial \mathcal{B}}\right)\right\} .
$$

By the concavity of $u$, we have

$$
0<u^{\prime}(\hat{q})<\frac{u(\hat{q})-u(q)}{\hat{q}-q}<u^{\prime}(q) .
$$


Hence, it holds that

$$
\left[u^{\prime}(\hat{q})-u^{\prime}(q)\right] \frac{\partial \hat{q}}{\partial \mathcal{B}}<\left[u^{\prime}(\hat{q}) \frac{\partial \hat{q}}{\partial \mathcal{B}}-u^{\prime}(q) \frac{\partial q}{\partial \mathcal{B}}\right]-\frac{u(\hat{q})-u(q)}{\hat{q}-q}\left(\frac{\partial \hat{q}}{\partial \mathcal{B}}-\frac{\partial q}{\partial \mathcal{B}}\right)<\left[u^{\prime}(\hat{q})-u^{\prime}(q)\right] \frac{\partial q}{\partial \mathcal{B}} .
$$

When $\delta=\eta=0$, by Proposition 11 we have $\partial q / \partial \mathcal{B}=0, \partial \hat{q} / \partial \mathcal{B}>0$. Hence, we have

$$
\left[u^{\prime}(\hat{q}) \frac{\partial \hat{q}}{\partial \mathcal{B}}-u^{\prime}(q) \frac{\partial q}{\partial \mathcal{B}}\right]-\frac{u(\hat{q})-u(q)}{\hat{q}-q}\left(\frac{\partial \hat{q}}{\partial \mathcal{B}}-\frac{\partial q}{\partial \mathcal{B}}\right)<0 \text { when } \delta=\eta=0 .
$$

Therefore, if $\delta$ and $\eta$ are small, by continuity, we have

$$
\frac{\partial \varphi^{-1}}{\partial \mathcal{B}}<0 ; \text { i.e., } \frac{\partial \varphi}{\partial \mathcal{B}}=-\varphi^{2} \frac{\partial \varphi^{-1}}{\partial \mathcal{B}}>0
$$

On the other hand, if $\delta=\eta=1$, by Proposition 11 we have $\partial q / \partial \mathcal{B}<0, \partial \hat{q} / \partial \mathcal{B}=0$. Hence, we have

$$
\left[u^{\prime}(\hat{q}) \frac{\partial \hat{q}}{\partial \mathcal{B}}-u^{\prime}(q) \frac{\partial q}{\partial \mathcal{B}}\right]-\frac{u(\hat{q})-u(q)}{\hat{q}-q}\left(\frac{\partial \hat{q}}{\partial \mathcal{B}}-\frac{\partial q}{\partial \mathcal{B}}\right)>0 \text { when } \delta=\eta=1 .
$$

Therefore if $\delta$ and $\eta$ are large, by continuity, we have

$$
\frac{\partial \varphi^{-1}}{\partial \mathcal{B}} \geq 0 ; \text { i.e., } \frac{\partial \varphi}{\partial \mathcal{B}}=-\varphi^{2} \frac{\partial \varphi^{-1}}{\partial \mathcal{B}} \leq 0 .
$$

Note that if $\eta=1$, then $\partial \varphi / \partial \mathcal{B}=0$ independent of $\delta$.

Proof of Proposition 15. In the type-I equilibrium, by differentiating equation (22) with respect to $\gamma$, we have

$$
\frac{\partial q}{\partial \gamma}=\frac{1}{\beta(1-n)(1-\delta \eta) u^{\prime \prime}(q)}<0
$$

In the type-II equilibrium, by differentiating equation (28) with respect to $\gamma$, we have

$$
\frac{1}{2} \frac{\partial \hat{q}}{\partial \gamma}=\frac{\partial q}{\partial \gamma}=\frac{1}{\beta(1-n)} \frac{1}{2 \delta u^{\prime \prime}(2 q)+(1-\delta \eta) u^{\prime \prime}(q)}<0 .
$$

For the type-III equilibrium, we show first that

$$
\frac{\partial \hat{q}}{\partial \gamma} \frac{\partial q}{\partial \gamma}>0
$$

By differentiating equation (33) with respect to $\gamma$, we have

$$
\mathcal{B} \frac{\partial q}{\partial \gamma}=\left[(1-\eta) u^{\prime}(\hat{q})+\eta\right] \frac{\partial \hat{q}}{\partial \gamma}-\left[(1-\eta) u^{\prime}(q)+\eta\right] \frac{\partial q}{\partial \gamma},
$$


so that

$$
\left[(1-\eta) u^{\prime}(\hat{q})+\eta\right] \frac{\partial \hat{q}}{\partial \gamma}=\left[(1-\eta) u^{\prime}(q)+\eta+\mathcal{B}\right] \frac{\partial q}{\partial \gamma} .
$$

Since $(1-\eta) u^{\prime}(\hat{q})+\eta>0$ and $\mathcal{B}>0$, we have $\frac{\partial \hat{q}}{\partial \gamma} \frac{\partial q}{\partial \gamma} \geq 0$. It is clear that $\frac{\partial \hat{q}}{\partial \gamma} \frac{\partial q}{\partial \gamma} \neq 0$ from the equations (34) and (55). Now, we show that $\partial \hat{q} / \partial \gamma<0$ and $\partial q / \partial \gamma<0$ by means of a contradiction. Suppose the contrary is true; i.e., we assume that

$$
\frac{\partial \hat{q}}{\partial \gamma}>0, \quad \frac{\partial q}{\partial \gamma}>0
$$

Note, by (54), that this case is only possible if " $\partial \hat{q} / \partial \gamma<0$ and $\partial q / \partial \gamma<0$ " are not true. By differentiating equation (34) with respect to $\gamma$, we have

$$
\begin{aligned}
\frac{1}{\beta(1-n)}= & \delta u^{\prime \prime}(\hat{q}) \frac{\partial \hat{q}}{\partial \gamma} \frac{\eta+(1-\eta) u^{\prime}(q)}{\eta+(1-\eta) u^{\prime}(\hat{q})}+\delta u^{\prime}(\hat{q}) \frac{(1-\eta) u^{\prime \prime}(q) \frac{\partial q}{\partial \gamma}\left[\eta+(1-\eta) u^{\prime}(\hat{q})\right]}{\left[\eta+(1-\eta) u^{\prime}(\hat{q})\right]^{2}} \\
& -\delta u^{\prime}(\hat{q}) \frac{(1-\eta) u^{\prime \prime}(\hat{q}) \frac{\partial \hat{q}}{\partial \gamma}\left[\eta+(1-\eta) u^{\prime}(q)\right]}{\left[\eta+(1-\eta) u^{\prime}(\hat{q})\right]^{2}}+(1-\delta) u^{\prime \prime}(q) \frac{\partial q}{\partial \gamma}
\end{aligned}
$$

Hence,

$$
\begin{aligned}
0<\frac{\left[\eta+(1-\eta) u^{\prime}(\hat{q})\right]^{2}}{\beta(1-n)}= & \delta u^{\prime \prime}(\hat{q}) \frac{\partial \hat{q}}{\partial \gamma}\left[\eta+(1-\eta) u^{\prime}(q)\right]\left[\eta+(1-\eta) u^{\prime}(\hat{q})\right] \\
& +\delta u^{\prime}(\hat{q})(1-\eta) u^{\prime \prime}(q) \frac{\partial q}{\partial \gamma}\left[\eta+(1-\eta) u^{\prime}(\hat{q})\right] \\
& -\delta u^{\prime}(\hat{q})(1-\eta) u^{\prime \prime}(\hat{q}) \frac{\partial \hat{q}}{\partial \gamma}\left[\eta+(1-\eta) u^{\prime}(q)\right] \\
& +(1-\delta) u^{\prime \prime}(q) \frac{\partial q}{\partial \gamma}\left[\eta+(1-\eta) u^{\prime}(\hat{q})\right]^{2} \\
= & \delta u^{\prime \prime}(\hat{q}) \frac{\partial \hat{q}}{\partial \gamma}\left[\eta+(1-\eta) u^{\prime}(q)\right] \eta \\
& +\delta u^{\prime}(\hat{q})(1-\eta) u^{\prime \prime}(q) \frac{\partial q}{\partial \gamma}\left[\eta+(1-\eta) u^{\prime}(\hat{q})\right] \\
& +(1-\delta) u^{\prime \prime}(q) \frac{\partial q}{\partial \gamma}\left[\eta+(1-\eta) u^{\prime}(\hat{q})\right]^{2}<0
\end{aligned}
$$

which is a contradiction. Therefore, we have

$$
\frac{\partial \hat{q}}{\partial \gamma}<0, \quad \frac{\partial q}{\partial \gamma}<0
$$


TABle A.1: Data Source

\begin{tabular}{ll}
\hline \hline Description & Identifier \\
\hline 3-month T-Bill rate & TB3MS \\
Consumer price index & CPIAUCSL_PC1 \\
M1 money stock & M1 \\
Nominal GDP & GDP \\
\hline
\end{tabular}

\section{A.2 Data Source}

All the data that we used for the calibration is downloadable from the Federal Reserve Bank of St. Louis FRED database, except for the outstanding T-Bills, which we obtained from the Treasury Bulletin. Table A.1 gives a brief overview of the respective identifiers.

Since the outstanding T-Bills are only available at a yearly frequency, we assumed that the outstanding amount remains unchanged within a year.

\section{References}

[1] Andolfatto, D., 2010, "Essential Interest-Bearing Money", Journal of Economic Theory, 145, 1319-1602.

[2] Andolfatto, D., 2011, "A Note on the Societal Benefits of Illiquid Bonds", Canadian Journal of Economics, 44, 133-147.

[3] Andolfatto, D., Martin, F., and Zhang, 2015, "Rehypothecation and Liquidity", working paper, Federal Reserve Bank of St. Louis.

[4] Ashcraft, A. B., and Duffie, D., 2007, "Systematic Illiquidity in the Federal Funds Market", American Economic Review, 97, 221-225.

[5] Aruoba, B., Rocheteau, G., and Waller, C., 2007, "Bargaining and the Value of Money", Journal of Monetary Economics, 54, 2636-2655.

[6] Berentsen, A., Camera, G., and Waller, C., 2007, "Money, Credit and Banking", Journal of Economic Theory, 135, 171-195.

[7] Berentsen, A., Huber, S., and Marchesiani, A., 2014, "Degreasing the Wheels of Finance", International Economic Review, 55, 735-763.

[8] Berentsen, A., Huber, S., and Marchesiani, A., 2016, "The Societal Benefit of a Financial Transaction Tax", European Economic Review, 89, 303-323.

[9] Berentsen, A., Menzio, G., and Wright, R., 2011, "Inflation and Unemployment in the Long Run", American Economic Review, 101, 371-398. 
[10] Berentsen, A., and Waller, C., 2011, "Outside Versus Inside Bonds: A Modigliani-Miller Type Result for Liquidity Constrained Economies", Journal of Economic Theory, 146, 18521887.

[11] Bethune, Z., Rocheteau, G., and Rupert, P. 2015, "Aggregate Unemployment and Household Unsecured Debt", Review of Economic Dynamics, 18, 77-100.

[12] Boel, P., and Waller, C., 2015, "On the Theoretical Efficacy of Quantitative Easing at the Zero Lower Bound", working paper, Federal Reserve Bank of St. Louis.

[13] Chun, Y., and Thomson, W., 1988, "Monotonicity properties of bargaining solutions when applied to economics", Mathematical Social Sciences, 15, 11-27.

[14] Dominguez, B., and Gomis-Porqueras, P., 2016, "The Effects of Secondary Markets and Unsecured Credit on Inflation Dynamics", MPRA Paper 7506.

[15] Duffie, D., Gârleanu, N., and Pederson, L. H., 2005, "Over-the-Counter Markets", Econometrica, 73, 1815-1847.

[16] Duffie, D., Gârleanu, N., and Pederson, L. H., 2008, "Valuation in Over-the-Counter Markets", Review of Financial Studies, 20, 1865-1900.

[17] Ferraris, L., and Watanabe, M., 2008, "Collateral secured loans in a monetary economy", Journal of Economic Theory, 143, 405-424.

[18] Forsythe, R., Horowith, J. L., Savin, N. E., and Sefton, M., 1994, "Fairness in Simple Bargaining Experiments", Games and Economic Behavior, 6, 347-369.

[19] Geromichalos, A., Licari, J., and Lledo, J. S., 2007, "Asset Prices and Monetary Policy", Review of Economic Dynamics, 10, 761-779.

[20] Geromichalos, A., and Herrenbrueck, L., 2016a, "Monetary Policy, Asset Prices, and Liquidity in Over-the-Counter Markets", Journal of Money, Credit and Banking, 28, 35-79.

[21] Geromichalos, A., and Herrenbrueck, L., 2016b, "The Strategic Determination of the Supply of Liquid Assets," working paper.

[22] Geromichalos, A., and Herrenbrueck, L., 2017, "A Tractable Model of Indirect Asset Liquidity," Journal of Economic Theory, 168, 252-260.

[23] Gertler, M., and Karadi, P., 2013, "QE 1 vs. 2 vs. 3...: A Framework for Analyzing LargeScale Asset Purchases as a Monetary Policy Tool", International Journal of Central Banking, 9, 5-53.

[24] Gomis-Porqueras, P., and Sanches, D., 2013, "Optimal Monetary Policy in a Model of Money and Credit", Journal of Money, Credit and Banking, 45, 701-730. 
[25] Greenwald, B., and Stiglitz, J., 1986, "Externalities in Economies with Imperfect Information and Incomplete Markets", Quarterly Journal of Economics, 101, 229-264.

[26] Gu, C., Mattesini, F., Monnet, C., and Wright, R., 2013, "Endogenous Credit Cycles", Journal of Political Economy, 121, 940-65.

[27] He, C., Wright, R., and Zhu, Y., 2015, "Housing and Liquidity", Review of Economic Dynamics, 18, 435-455.

[28] Herrenbrueck, L., 2014, "QE and the Liquidity Channel of Monetary Policy", working paper, Simon Fraser University.

[29] Hu, T., and Rocheteau, G., 2013, "On the Coexistence of Money and Higher Return Assets", Journal of Economic Theory, 148, 2520-2560.

[30] Huber, S., and Kim, J., 2016, "Centralized Trading of Corporate Bonds", working paper (first version 2015), University of Zürich.

[31] Jacquet, N. L., and Tan, S., 2012, "Money and Asset Prices with Uninsurable Risk", Journal of Monetary Economics, 59, 784-797.

[32] Kalai, E., 1977, "Proportional Solutions to Bargaining Situations: Interpersonal Utility Comparisons", Econometrica, 45, 1623-1630.

[33] Kiyotaki, N., and Wright, R., 1989, "On Money as a Medium of Exchange", Journal of Political Economy, 97, 927-954.

[34] Kocherlakota, N., 1998, "Money Is Memory", Journal of Economic Theory, 81, 232-251.

[35] Kocherlakota, N., 2003, "Societal Benefits of Illiquid Bonds", Journal of Economic Theory, 108, 179-193.

[36] Krishnamurthy, A., 2002, "The Bond/Old-Bond Spread", Journal of Financial Economics, 66, 463-506.

[37] Lagos, R., 2010a, "Some Results on the Optimality and Implementation of the Friedman Rule in the Search Theory of Money", Journal of Economic Theory, 145, 1508-1524.

[38] Lagos, R., 2010b, "Asset Prices and Liquidity in an Exchange Economy", Journal of Monetary Economics, 57, 913-930.

[39] Lagos, R., 2011, "Asset Prices, Liquidity, and Monetary Policy in an Exchange Economy", Journal of Money, Credit and Banking, 43, 521-552.

[40] Lagos, R., and Rocheteau, G., 2008, "Money and Capital as Competing Media of Exchange", Journal of Economic Theory, 142, 247-258. 
[41] Lagos, R., and Rocheteau, G., 2009, "Liquidity in Asset Markets with Search Frictions", Econometrica, 77, 403-426.

[42] Lagos, R., Rocheteau, G., and Weill, P., 2011, "Crises and Liquidity in Over-the-Counter Markets", Journal of Economic Theory, 146, 2169-2205.

[43] Lagos, R., Rocheteau, G., and Wright, R., 2015, "Liquidity: A New Monetarist Perspective", Journal of Economic Literature, forthcoming.

[44] Lagos, R., and Wright, R., 2005, "A Unified Framework for Monetary Theory and Policy Evaluation", Journal of Political Economy, 113, 463-484.

[45] Lagos, R., and Zhang, S., 2015, "Monetary Exchange in Over-the-Counter Markets: A Theory of Speculative Bubbles, the Fed Model, and Self-fulfilling Liquidity Crises", working paper (first version 2014).

[46] Lester, B., Postlewaite, A., and Wright, R., 2012, "Liquidity, Information, Asset Prices and Monetary Policy", Review of Economic Studies, 79, 1209-1238.

[47] Li, Y., and Li, Y., 2012, "Liquidity, Asset Prices, and Credit Constraints", working paper, National Taiwan University.

[48] Mattesini, F., and Nosal, E., 2016, "Liquidity and asset prices in a monetary model with OTC asset markets", Journal of Economic Theory, 164, 187-217.

[49] Nosal, E., and Rocheteau, G., 2011, "Money, Payments, and Liquidity", MIT Press.

[50] Nosal, E., and Rocheteau, G., 2013, "Pairwise Trades, Asset Prices, and Monetary Policy", Journal of Economic Dynamics and Control, 37, 1-17.

[51] Pontrandolfo, F., 2015, "LQA Liquidity Assessment Tool", Bloomberg L.P.

[52] Rocheteau, G., and Weill, P. O., 2011, "Liquidity in Frictional Asset Markets," Journal of Money, Credit and Banking, 43, 261-282.

[53] Rocheteau, G., and Wright, R., 2005, "Money in Competitive Equilibrium, in Search Equilibrium, and in Competitive Search Equilibrium", Econometrica, 73, 175-202.

[54] Rocheteau, G., and Wright, R., 2013, "Liquidity and Asset Market Dynamics", Journal of Monetary Economics, 60, 275-294.

[55] Rocheteau, G., Wright, R., and Xiao, S. X., 2015, "Open Market Operations", working paper.

[56] Roth, A. E., Prasnikar, V., Okuno-Fujiwara, M., Zamir, S., 1991, "Bargaining and Market Behaviour in Jerusalem, Ljublijana, Pittsburgh, and Tokyo: An Experimental Study", American Economic Review, 81, 1068-1095. 
[57] Shi, S., 2006, "Viewpoint: A Microfoundation of Monetary Economics", Canadian Journal of Economics, 39, 643-688.

[58] Shi, S., 2008, "Efficiency Improvement from Restricting the Liquidity of Nominal Bonds", Journal of Monetary Economics, 55, 1025-1037.

[59] Slonim, R., and Roth, A. E., 1998, "Learning in High Stakes Ultimatum Games: An Experiment in the Slovak Republic", Econometrica, 66, 569-596.

[60] Trejos, A., and Wright, R., 2016, "Search-based models of money and finance: An integrated approach", Journal of Economic Theory, 164, 10-31.

[61] Wallace, N., 1981, "A Modigliani-Miller Theorem for Open-Market Operations", American Economic Review, 71, 267-274.

[62] Wallace, N., 2001, "Whither Monetary Economics?", International Economic Review, 42, 847-869.

[63] Williamson, S., 2012, "Liquidity, Monetary Policy, and the Financial Crisis: A New Monetarist Approach", American Economic Review, 102, 2570-2605.

[64] Williamson, S., 2015, "Central Bank Purchases of Private Assets", working paper, Federal Reserve Bank of St. Louis.

[65] Williamson, S., 2016, "Scarce Collateral, the Term Premium, and QE", Journal of Economic Theory, 164, 136-165.

[66] Williamson, S., and Wright, R., 2010 "New Monetarist Economics: Models", in B. Friedman and M. Woodford (eds.) Handbook of Monetary Economics, Volume II, Amsterdam: NorthHolland. 